\title{
Rheology and scaling behavior of cascading granular flows in rotating drums
}

Luisa Fernanda Orozco, ${ }^{1,2}$, a) Jean-Yves Delenne, ${ }^{3}$ Philippe Sornay, ${ }^{4}$ and Farhang Radjai $^{2}$

${ }^{1)}$ CEA, DEN, DEC, SA3E, LCU, 13108 Saint Paul les Durance, France

${ }^{2)}$ LMGC, CNRS, University of Montpellier, France

${ }^{3)}$ IATE, INRA, CIRAD, Montpellier SupAgro, University of Montpellier,

France

${ }^{4)}$ CEA, DES, IRESNE, DEC, SA3E, LCU, F-13108 Saint-Paul-lez-Durance,

France

Mixing, agglomerating and milling granular materials within a rotating drum is often performed in the cascading flow regime, however the scaling behavior of these industrial applications remains poorly understood. It involves both centrifugal forces and an inertial surface flow with a curved profile. By means of discrete element simulations, we investigate the rheology of cascading flows in rotating drums as a function of drum size, rotation speed and filling degree. We find that the surface profile, described by the ratio between steepest descent slope and an average slope, is strongly correlated with flow variables such as active layer thickness, contact force variability and wall slip. We show that the flow variables cannot be scaled by Froude number alone, but are instead nicely scaled by a dimensionless parameter that combines the Froude number with other system parameters. This scaling works even for small drums and low filling degrees where finite size effects prevail and the slippage of particles at the drum wall considerably affects the cascading flow at the free surface. The observed correlation between this parameter and contact force fluctuations suggests that it may also be a relevant upscaling parameter for milling and agglomeration in rotating drums.

Keywords: Granular materials, rotating drum, upscaling, Discrete Element Method, Contact Dynamics Method

\footnotetext{
a)luisa.orozco@umontpellier.fr
} 


\section{INTRODUCTION}

Rotating drums are extensively used in applications such as granulation, grinding and mixing of granular materials ${ }^{1-4}$. They have also been used for the investigation of steady-state granular

flows $5,6,8-11$. However, the dynamics and rheology of granular materials in rotating drums are complex mainly because of the inhomogeneous flow of particles combining upward rigid-body motion of the particles at the drum wall, downward bulk flow and free surface dynamics. The drum flow depends on the rotation speed, drum internal radius, drum width and filling height. The effects of these parameters can be described in terms of the free surface profile, bulk flow behavior and micromechanical variables such as contact forces and displacement fields at the particle scale. The Froude number $\mathrm{Fr}=R \omega^{2} / g$, where $g$ is the gravity acceleration, $\omega$ is rotation speed and $R$ is drum radius, was suggested for the scale-up of measurable variables from the laboratory to the pilot or industrial scales ${ }^{12,13}$. However, several studies suggest that the Froude number is insufficient for scaling purposes as it does not account for finite-size effects and filling degree that affect features such as free surface profile, thickness of the active flowing layer and surface velocity ${ }^{14-18}$.

Taberlet et al. ${ }^{15}$ found that the differences of surface profiles could be linked to the friction with the end-walls, and by considering mass conservation they proposed a scaling that includes the drum width and radius. Pignatel et al. ${ }^{20}$ found that the inertial number ${ }^{19}$ should be modified in order to achieve a better scaling of the flowing layer thickness in rotating drums filled with dry and wet granular materials. Iwasaki et al. ${ }^{16}$ performed experiments and numerical simulations on drums of four different diameters and proposed a scaling based on the dissipated power. They found that the energy distribution function and the final particle size distribution in a grinding process were the same for the four drums when using their scaling. However, most reported scaling laws concern mainly the rolling regime (see below), low values of rotation speed or small particle size ratios, and there is presently no general agreement on the upscaling of rotating drums ${ }^{1}$.

A major issue for a better understanding of the rotating drums is the granular rheology at intermediate rotation speeds. Different flow regimes occur in rotating drums depending strongly on the rotation speed but also on drum size and filling ratio. A common classification includes successively the sliding (or slumping), surging, rolling, cascading, cataracting and centrifuging regimes as the rotation speed increases ${ }^{21}$. The first two regimes are characterized by the movement of the particles as a block that slides or oscillates on the drum wall. In the rolling regime, a shear flow is formed at the free surface, which is flat with a dynamic slope, and inertial 
effects govern the flow behavior. This regime is conveniently described by the inertial rheology applied to the free surface flow ${ }^{22}$ by accounting for the cylindrical geometry ${ }^{23}$. In the cascading regime, the free surface is curved and the particle dynamics along the steepest descent is highly collisional. Finally, in the cataracting regime, the particles ejected from the upstream end of the free surface undergo ballistic motions and collide with the downstream part of the free surface. The cascading and cataracting regimes provide suitable operational conditions for industrial mills and granulators ${ }^{21,24}$. In particular, the active flowing layer thickness is an important quantity for mixing, grinding, thermal and convection properties ${ }^{19,20,25-30}$. Nevertheless, these regimes remain poorly understood.

In this paper, we present a parametric study of granular flow in the cascading regime in rotating drums by means of Discrete-Element simulations for a broad range of the values of rotation speed, drum radius, drum width, flow thickness and particle diameter. We consider the shape of the free surface in terms of its average slope and steepest slope, flow thickness, particle slip at the drum walls, strain-rate field, contact force fluctuations. We also introduce a dimensionless upscaling parameter that combines different system parameters and conveniently describes the flow behavior in the cascading regime.

In the following, we first introduce in Section II the numerical method, sample construction and simulation parameters. The, in Section III, we present the velocity profiles and related kinematic fields such as local strain rates and granular temperature. This allows us to define the average flow parameters that will be used for the scaling of the cascading flow. In Section IV, we characterize the free surface profiles and the influence of system parameters such as Froude number and drum size. Section $\mathrm{V}$ is devoted to the investigation of contact forces and their probability density functions, as well as the correlation between force fluctuations and free surface shape. In Section VI, we introduce a scale parameter that accounts for all flow characteristics as a function of the investigated system parameters, including Froude number, drum size, particle size, and filling degree. Finally, in Section VII, we discuss the most salient results of this work and suggested directions of research. 


\section{NUMERICAL METHOD AND PROCEDURES}

\section{A. Contact Dynamics Method}

The numerical simulations were carried out by means of the Contact Dynamics Method $(\mathrm{CDM})^{31,32}$, which has been proven to be adequate to simulate the particles dense flows ${ }^{33,34}$. This is an implicit variant of the discrete element method (DEM) in which an iterative Gauss-Seidel algorithm is used for the time-stepping integration of the equations of motion for all particles subjected to frictional contact interactions. In contrast to the explicit approach, in which overlaps between particles are allowed and penalized by repulsive or viscous forces, the CDM is based on two contact laws: 1) the Signorini condition, defined as a complementarity relation between normal contact velocity $u_{n}$ and normal force $f_{n}$, and 2) the Coulomb dry friction law, defined as a complementarity relation between the sliding velocity $u_{t}$ and the friction force $f_{t}$. These contact laws are represented as graphs in Fig. 1. A "complementarity relation" expresses the property that the relation between a pair of variables cannot be reduced to a mono-valued function and the two variables cannot be nonzero at the same time ${ }^{35}$. The particle velocities and contact forces are calculated simultaneously at each time step by an iterative process for the contact network defined from particle positions at the beginning of the time step and used to update the particle positions. Due to the implicit integration scheme used, the method is unconditionally stable, allowing thus for large time steps as compared to the explicit methods. We used the LMGC90 software for all the simulations analyzed in this paper.

\section{B. Sample setup and boundary conditions}

We consider drums of different diameters $2 R$ and widths $W$ filled with monodisperse spheres of diameter $d=2 r$ and subjected to a constant rotation speed $\omega$; see Fig. 2. The filling degree is defined by the ratio $J=h_{0} / 2 R$, where $h_{0}$ is the thickness of the granular flow at the mid-chord point of the free surface at rest. Periodic boundary conditions were imposed along the drum axis so that the flow is invariant along the $y$ axis. The coefficient of friction between the particles and with drum walls was set to $\mu=0.4$, representing a typical value for most materials ${ }^{36,37}$. The normal and tangential coefficients of restitution were set to zero, corresponding to fully inelastic collisions. As dense frictional granular flows are insensitive to the value of restitution coefficient (except for values very close to 1 ), this choice is not critical for rotating drum simulations ${ }^{32,38}$. 


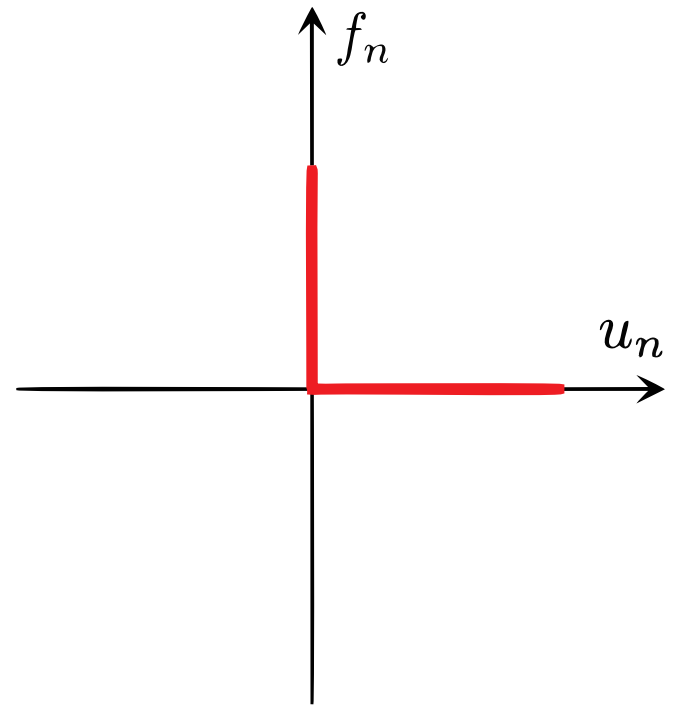

(a)

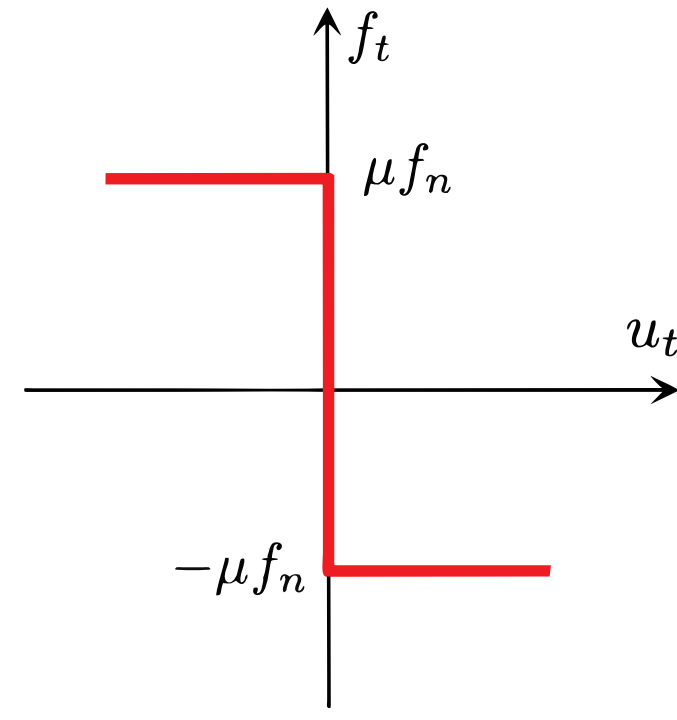

(b)

FIG. 1. Contact laws used in the contact dynamics method (CDM): a) Signorini relation between normal force $f_{n}$ and normal contact velocity $u_{n}$, b) Coulomb friction law as the relation between sliding velocity $u_{t}$ and friction force $f_{t}$, at a contact point between two particles.

The simulations were performed for a range of values of $\omega, J, R / r, h_{0} / 2 R$ and $W$, as shown in Table I. For most simulations, we used a drum width $W_{0}=0.05 \mathrm{~m}$. A few more simulations were performed for $W=2 W_{0}$ and $W=4 W_{0}$. Hence, in the following, the default width is $W_{0}$ unless explicitly mentioned. The default filling degree was set to $J=0.45$ except for a few simulations with $J=0.27$ and $J=0.38$, which will be explicitly mentioned. Most simulations were run for 10 seconds and more to allow the system to reach a steady flow state. The data analyzed in this paper, such as the free surface profiles, flow thickness and mean particle velocities are average values in this steady flow state. The focus of the investigations analyzed in this paper was the effect of drum size although we considered the influence of other system parameters. In order to single out the effect of drum size, we performed more specifically two sets of simulations. In the first set, the Froude number was kept constant at 0.8 and other parameters were changed whereas in the second set the rotation speed was kept at $\omega=5 \mathrm{rad} / \mathrm{s}$ and other parameters were varied. For most sets of parameter values considered in this paper, the steady-state flow is in the cascading regime. 
TABLE I. Simulation parameters

\begin{tabular}{llll}
\hline Parameter & Symbol & Value & Unit \\
\hline Number of particles & $N_{p}$ & \multicolumn{2}{l}{$[839 ; 37509]$} \\
Particle density & $\rho$ & 2200 & $\mathrm{~kg} / \mathrm{m}^{3}$ \\
Friction coefficient & $\mu$ & 0.4 & \\
Normal restitution coefficient & $e_{n}$ & 0 & \\
Tangential restitution coefficient & $e_{t}$ & 0 & \\
Time step & $\delta t$ & $1 \times 10^{-4}$ & $\mathrm{~s}$ \\
Gravity acceleration & $g$ & 9.81 & $\mathrm{~m} / \mathrm{s}^{2}$ \\
Particle diameter & $d$ & 8 & $\mathrm{~mm}$ \\
Rotation speed & $\omega$ & {$[3.43 ; 10.23] \mathrm{rad} / \mathrm{s}$} \\
Froude number & $\mathrm{Fr}$ & {$[0.19 ; 0.96]$} & \\
Drum diameter/particle diameter & $2 R / d$ & {$[18.75 ; 125]$} & \\
Drum width/particle diameter & $W / d$ & {$[6.25 ; 25]$} & \\
Filling degree & $J=h_{0} / 2 R[0.27 ; 0.45]$ & \\
\hline
\end{tabular}

\section{PARTICLE VELOCITY FIELDS}

Figure 3 displays snapshots of the particle velocity vectors in four drums of different diameters but with the same value of the Froude number Fr and filling degree $J=h_{0} / 2 R$. The velocities are normalized by $\omega R$ in each case. The largest velocities are located at the free surface and in the vicinity of the drum wall. They increase in magnitude with drum size despite the constant value of Fr, meaning that, as we shall see in more detail below, the Froude number is not the only control parameter of granular flow in the drum geometry. We also observe that the shape of the free surface is increasingly curved as drum diameter increases.

Figure 4(a) shows velocity profiles along the secant slope of the free surface $\theta_{m}$ at the center of the drum for different values of drum size $R / r$ at constant rotation speed $\omega=5 \mathrm{rad} / \mathrm{s}$. The secant slope is defined by joining the uppermost point of the free surface to its lowermost point. It represents an average slope of the free surface. The profiles are basically nonlinear but get increasingly close to a linear profile for larger drums in exception to the layers close to the drum wall (with a nearly exponential increase) and at the free surface where the velocities are those of 

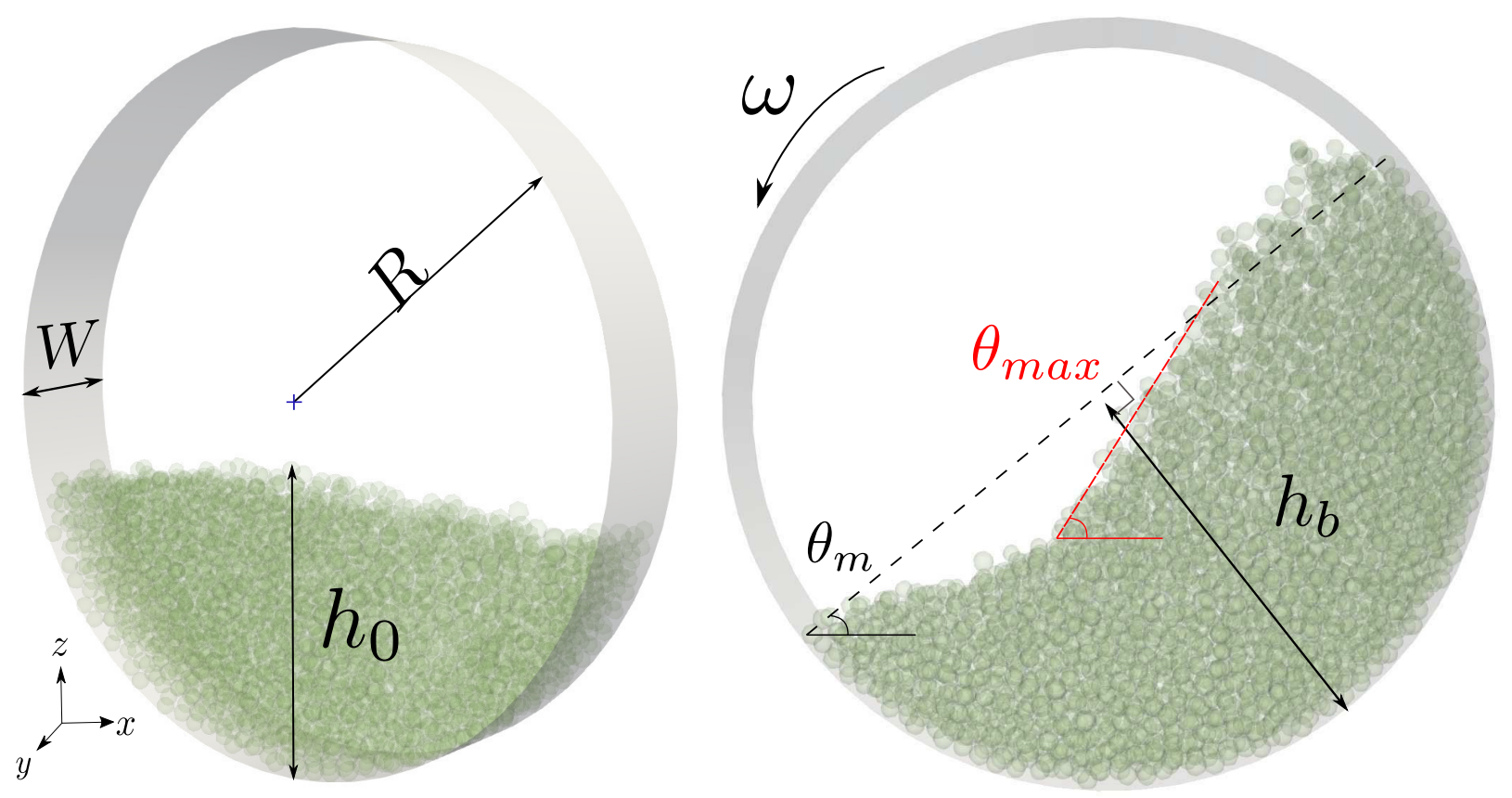

FIG. 2. Geometrical parameters of granular flow in a rotating drum in the initial state (a) and in steady flow (b).

cascading particles with values larger than $\omega R$. The locus of particles of vanishing velocity is the borderline between upward flow of the particles by drum rotation in the vicinity of the drum wall and downward flow (active layer) close to the free surface. We see that the thickness $h_{a}$ of the active layer increases with drum diameter.

Another feature observed in Fig. 4(a) is that the particles in contact with the drum wall do not generally follow the drum rotation, implying their partial slip against the drum wall. The amount of wall slip declines with increasing drum size. The particle velocities at the drum wall cannot flow faster than $\omega R$, but we observe larger values in Fig. 4 since the velocities are projected on the secant slope, which increases with drum diameter. Previous studies of rotating drums have reported various velocity profiles measured at the mid-section, including linear profile as a function of depth ${ }^{29,39-41}$, bi-linear ${ }^{42}$, linear-exponential ${ }^{7,43,44}$, and linear-parabolic ${ }^{45}$. However, in all those studies the system was mainly in the rolling regime while the profiles in our work belong to the cascading regime.

Figure 4(b) displays the velocity profiles at constant Froude number $(\mathrm{Fr}=0.8)$ and different values of drum size (implying different values of rotation speed $\omega[3.96,10.23] \mathrm{rad} / \mathrm{s}$ ). We see that, despite the same value of Froude number, the profiles depend on drum size although they are 


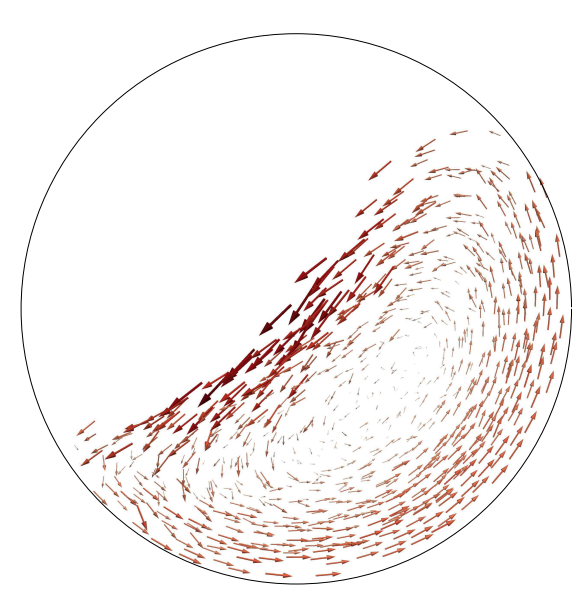

(a)

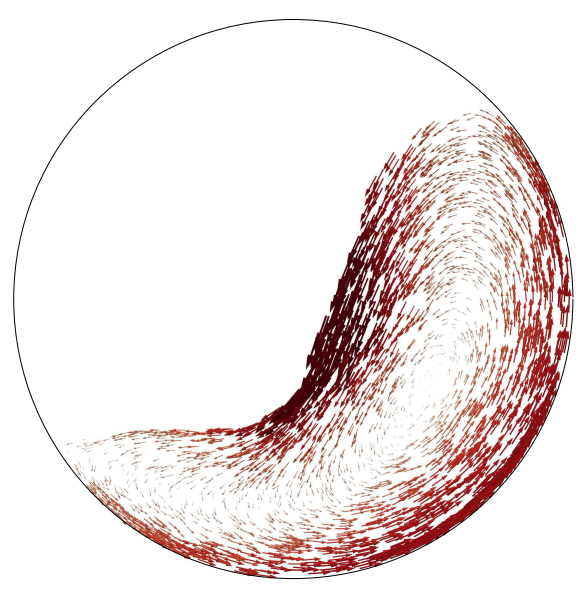

(c)

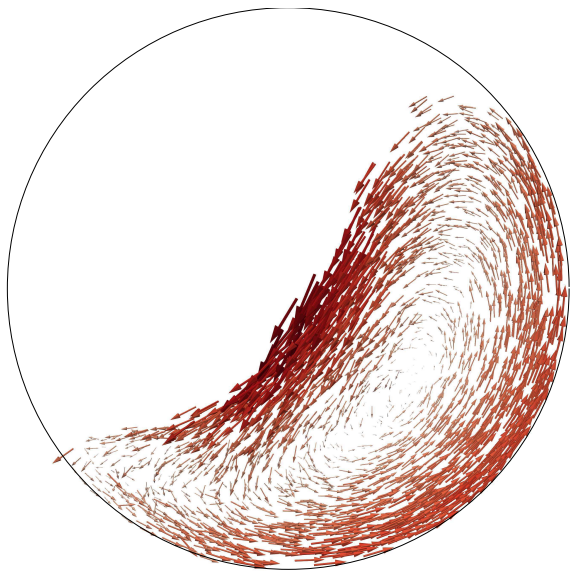

(b)

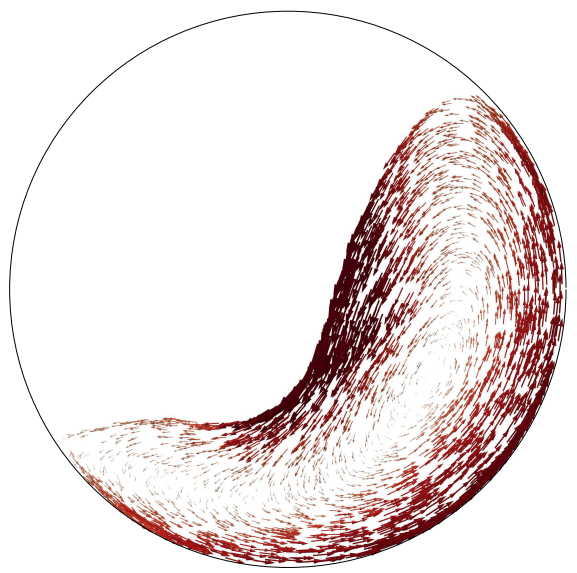

(d)

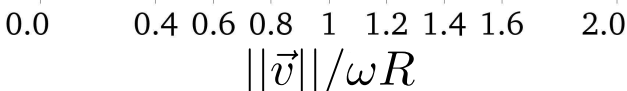

$\|\vec{v}\| / \omega R$

FIG. 3. Velocity vector fields in drums of different normalized sizes $R / r$ : a) 18.75 , b) 37.5, c) 62.5, d) 100 . The Froude number is $\mathrm{Fr}=0.8$ in all cases.

less scattered than in Fig. 4(a).

From the particle velocities and positions, it is possible to calculate the strain-rate tensor $\dot{\varepsilon}$ in the neighborhood of each particle $i$ using the following expression ${ }^{47}$ :

$$
\dot{\varepsilon}_{i}=\sum_{j}\left(\vec{v}_{i}-\vec{v}_{j}\right) \otimes \overrightarrow{n_{i j}} \frac{1}{\ell_{i j}}
$$

where $j$ denotes neighboring particles of particle $i$ within a distance $2 d=4 r, \overrightarrow{n_{i j}}$ is the unit vector normal to the line joining particles $i$ and $j, \ell_{i j}$ is the distance between their centers, and $\otimes$ is the dyadic product. Due to periodicity along $y$ direction, the strain-rate eigenvalues along $y$ direction 


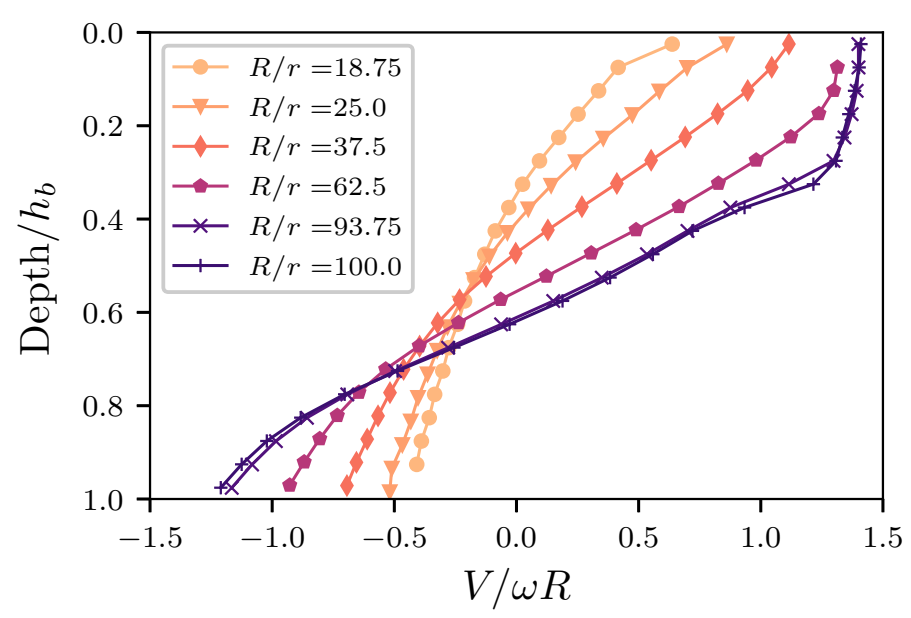

(a)

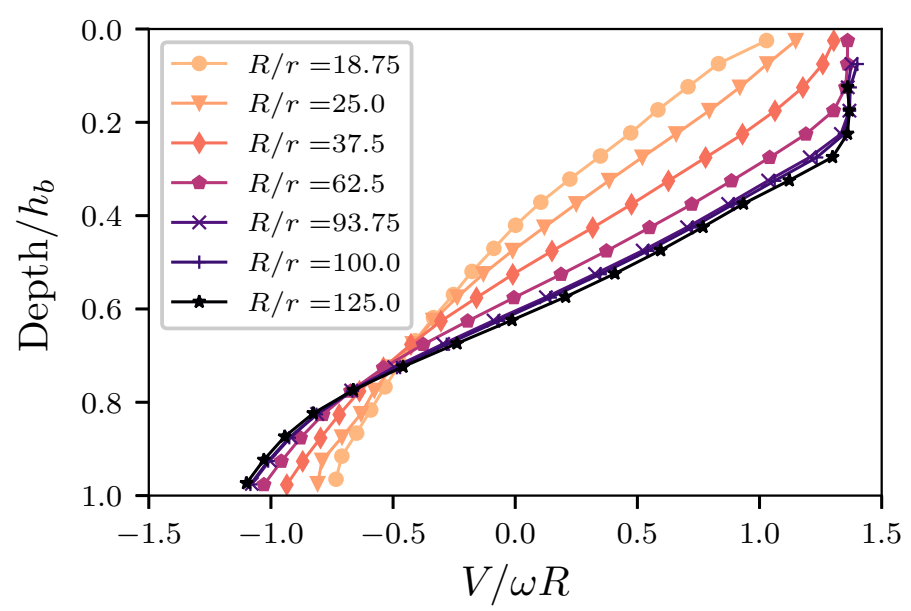

(b)

FIG. 4. Velocity profile at the center of the drum for different values of drum size $R / r$ at constant rotation speed $\omega$ (a) and at constant Froude number (b). The depth, measured in the $z$ direction, is normalized by the bed depth $h_{b}$. The velocity component $V$ along the mean free surface direction is normalized by $R \omega$.

vanish and only the plane strain rates on the $x z$ plane are relevant. The volumetric strain rate $\dot{\varepsilon}_{p}$, representing local volume change rate (positive for expansion and negative for contraction), and deviatoric strain rate $\dot{\varepsilon}_{q}$ can be computed from the eigenvalues $\dot{\varepsilon}_{1}$ and $\dot{\varepsilon}_{2}$ as follows:

$$
\dot{\varepsilon}_{p}=\dot{\varepsilon}_{1}+\dot{\varepsilon}_{2}, \dot{\varepsilon}_{q}=\dot{\varepsilon}_{1}-\dot{\varepsilon}_{2}
$$

Figures 5 and 6 display time-averaged fields of the volumetric and deviatoric strain rates, respectively, for four different drum diameters and a constant Froude number. We see that, while the average volume change is zero, the particles in the bulk of the bed show nearly no volume change whereas the dilating (positive values) and contracting (negative values) zones are located 


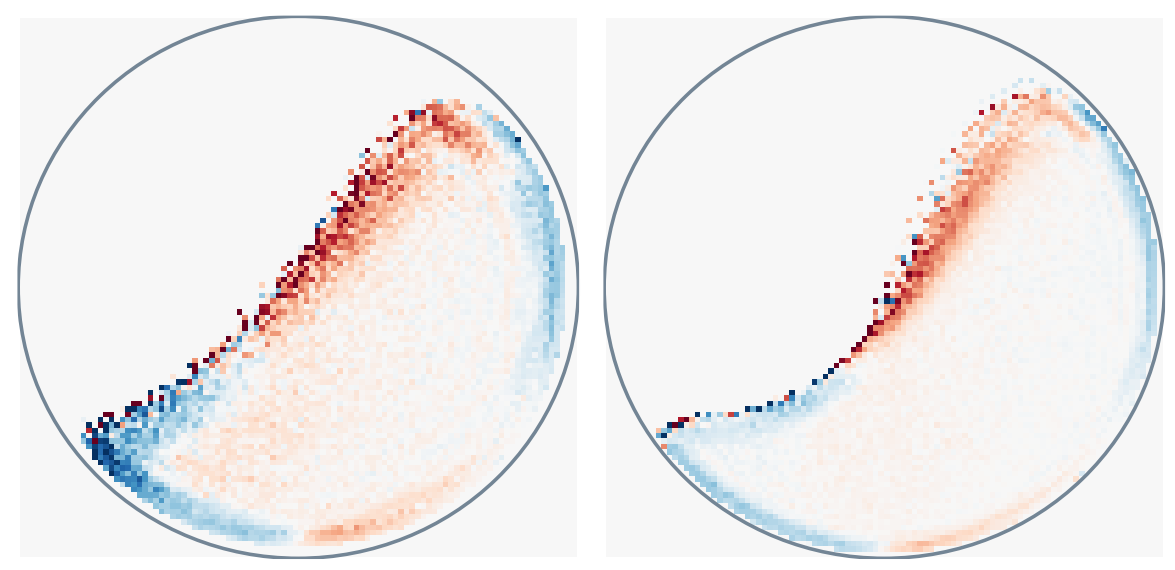

(a)

(b)
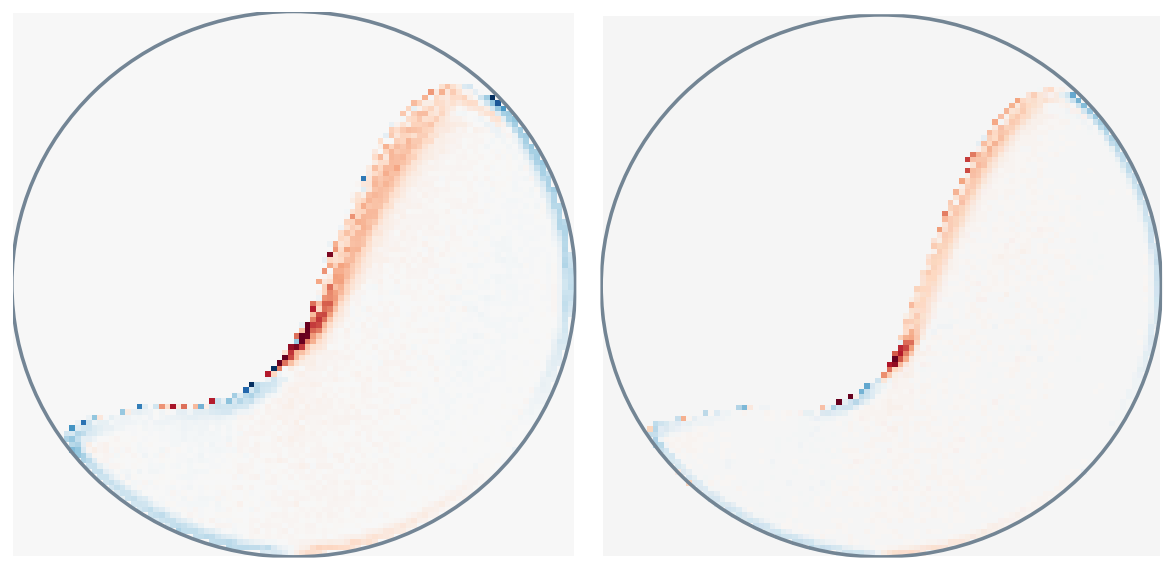

(c)

(d)

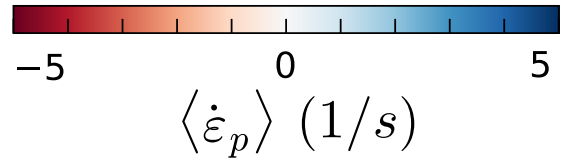

FIG. 5. Maps of local volume-change rates $\dot{\varepsilon}_{p}$ in drums of four different size ratios $R / r:$ a) 18.75 , b) 37.5 , c) 62.5 and d) 100 , for $\mathrm{Fr}=0.8$, averaged in time.

at the free surface and close to the drum wall over a few layers, respectively. The zones of intense shear rate $\dot{\varepsilon}_{q}$ are mainly located inside the cascade and at its toe where the particles collide with the granular bed after a cascading flow. This point moves progressively from the downstream of the free surface (at the wall) toward the center of the profile as drum size is increased. The cascading flow has a contracting behavior and thus contains more collisional than frictional interactions.

We also see a transition of the flow characteristics with increasing drum size. For the smallest drum, we observe a constant gradient of $\dot{\varepsilon}_{q}$ with the depth, as in a granular material flowing down an inclined plane. As the drum size is increased, the free surface develops two distinct parts: in the 


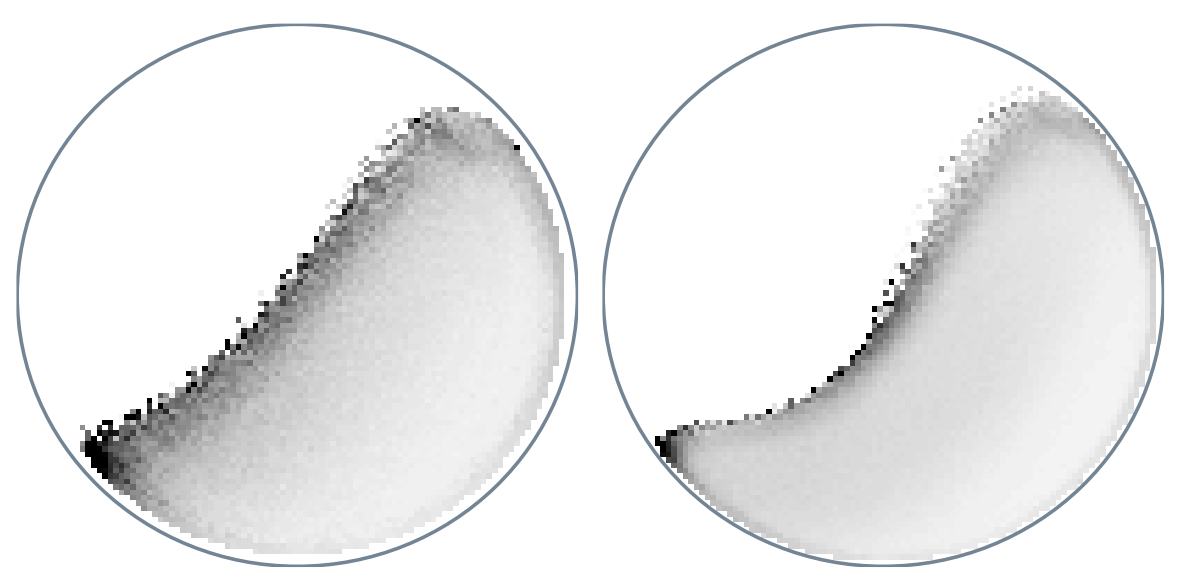

(a)

(b)

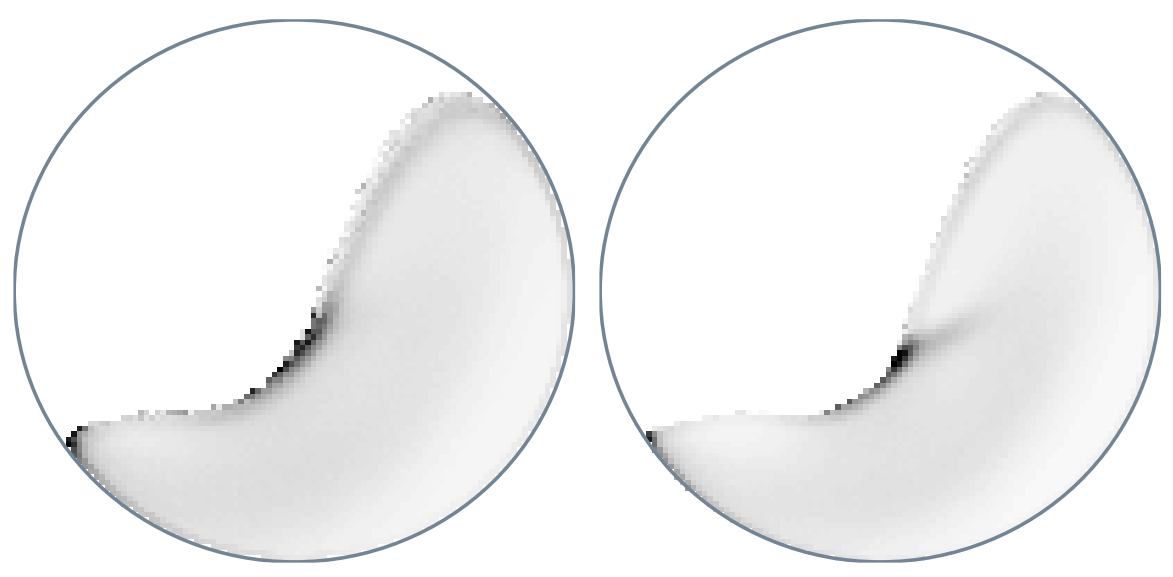

(c)

(d)

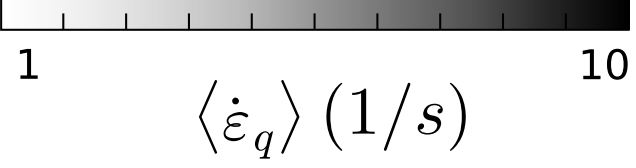

FIG. 6. Maps of local shear rates $\dot{\varepsilon}_{q}$ in drums of four different size ratios $R / r:$ a) 18.75 , b) 37.5 , c) 62.5 and d) 100 , for $\mathrm{Fr}=0.8$, averaged in time.

right part of the drum, the particles experience a cascading flow, and in the left part the particles undergo very low shearing and tend to follow drum rotation in transition from the active layer to the passive layer (upward flow). It is also remarkable that the shear strain is lower at the drum walls for larger drum size. This is consistent with the observation that the wall slip declines with increasing drum size, the particles near the wall following more closely (and rigidly) the drum rotation.

The active flowing layer on top of the granular bed is fed by the upward flow of particles driven 


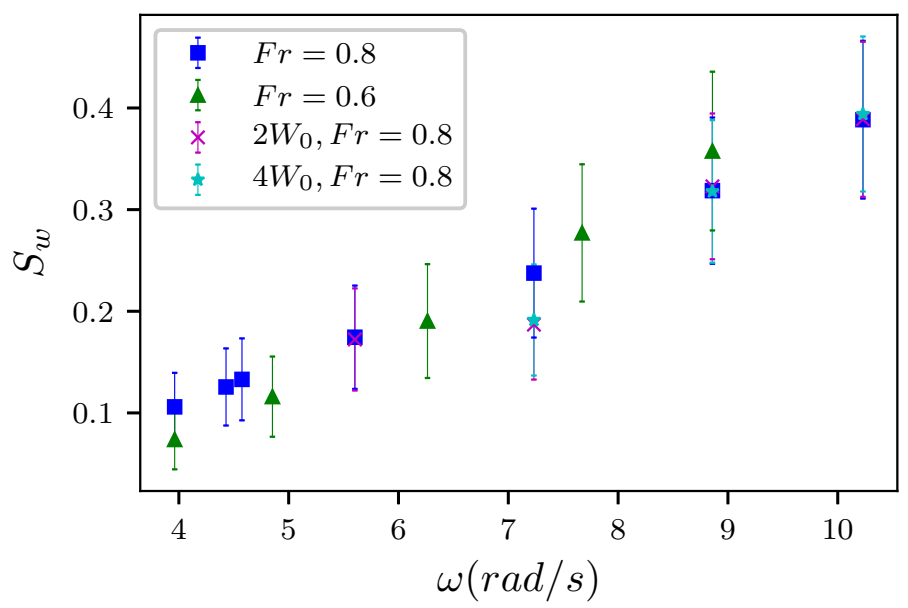

FIG. 7. Wall slip $S_{w}$ as a function of rotation speed $\omega$ for two values of Fr and three values of drum width $W$. The error bars reflect the standard deviations of slip velocities $V_{w}$.

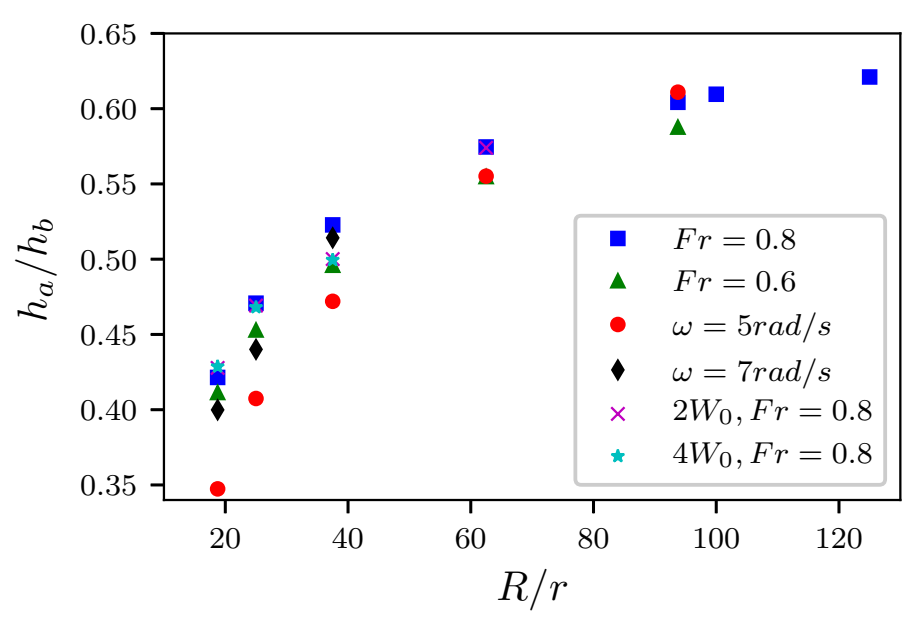

FIG. 8. The thickness $h_{a}$ of the active flowing layer normalized by flow thickness $h_{b}$ as a function of drum size $R / r$ for the tested range of system parameters.

by drum rotation. The feeding rate, i.e. the number of particles per unit time released from the upstream tip, depends both on the rotation speed and slip of the particles along the drum wall. We measured the time-averaged tangential speed $V_{w}$ of the particles in contact with the drum wall in the steady state. We consider a dimensionless slip parameter $S_{w}$ defined by

$$
S_{w}=1-\frac{V_{w}}{R \omega}
$$




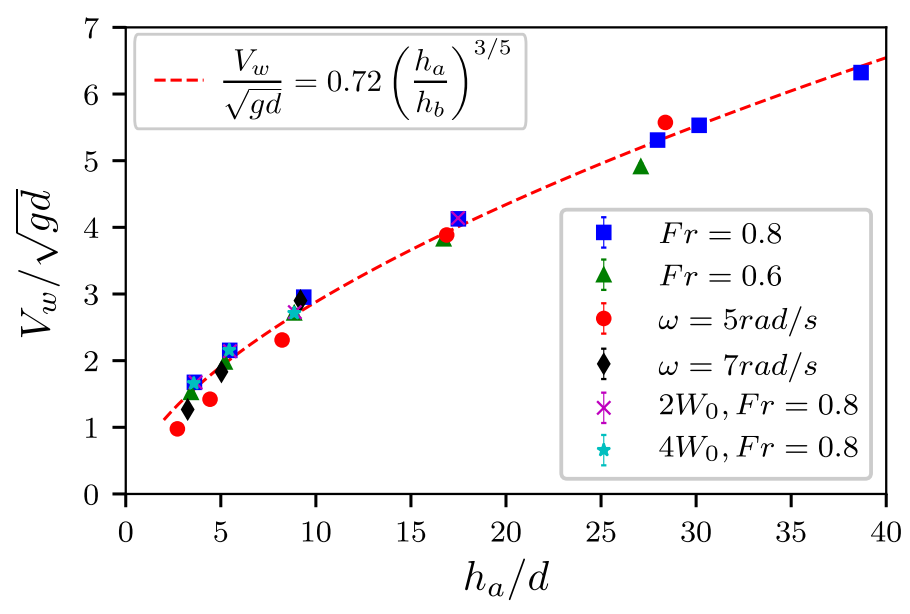

FIG. 9. Average speed $V_{w}$ of the particles at contact with the drum wall normalized by the characteristic velocity $\sqrt{g d}$ versus the thickness $h_{a}$ of the active flowing layer normalized by particle diameter $d$ for all simulations with different parameter values. The error bars representing the standard deviation are smaller than symbol size.

Figure 7 shows $S_{w}$ as a function of $\omega$ for $\mathrm{Fr}=0.8$ and $\mathrm{Fr}=0.6$, and three different values of the drum width $W$. We see that, up to statistical fluctuations, the wall slip is an increasing function of $\omega$. Note that, for a constant value of Fr, $R$ should decrease with increasing $\omega$. It is remarkable that, despite different values of Fr and $W$, all the data points define a single linear trend. The wall slip appears thus to be a finite size effect with increasing wall slip for smaller drum sizes.

We also measured the average thickness $h_{a}$ of the active layer at the center of the drum. Figure 8 shows the normalized active layer thickness $h_{a} / h_{b}$ as a function of $R / r$ for several values of Froude number or $\omega$ and drum width. The observed increase of $h_{a} / h_{b}$ with increasing drum size is consistent with the decrease of wall slip. Hence, the decrease of wall slip implies higher particle feed proportionally to $V_{w}$ at the upstream end of the free surface, leading to a thicker flowing layer as well as a more curved free surface shape. This relation is directly evidenced in Fig. 9 where $V_{w} / \sqrt{d g}$ is plotted against $h_{a} / d$ for all simulations. The data points show a good collapse, and they are well fit by a power law

$$
\frac{V_{w}}{\sqrt{d g}} \simeq 0.7\left(\frac{h_{a}}{d}\right)^{n},
$$

with $n \simeq 3 / 5$. We used the intrinsic scales ( $\sqrt{d g}$ and $d$ ) of the flow instead of $h_{b}$ and $\omega R$ since the relation between $V_{w}$ and $h_{a}$ reflects the conservation of the number of particles, and thus it 
applies to the absolute values of flow variables rather than their relative values. This relation with its exponent can be considered as an intrinsic feature of the cascading flow regime.

The particle scale processes such as particle fracture and mixing are generally governed by velocity fluctuations rather than mean velocities. The kinetic and inertial stresses arise from velocity fluctuations and they are modeled by introducing the granular temperature field $T$ defined as the mean square fluctuating velocity of particles at each point of the system:

$$
T=\left\langle v^{2}\right\rangle-\langle v\rangle^{2}
$$

Figure 10 displays the temperature fields normalized by $(R \omega)^{2}$ for four different drum sizes. As the volumetric and shear strain-rate fields reflect the local relative velocities of the particles, the granular temperature field as a scalar variable combines the volumetric and shear strain rates. The maps show increasing contrast for larger drum sizes with high temperatures in the downstream of the active layer. Its location moves from the endpoint of the free surface in smaller drums to the center of the flow in larger drums as also observed in shear-rate maps of Fig. 6.

\section{FREE SURFACE PROFILES}

The average free surface profiles are shown in Fig. 11(a) for different drum sizes and a constant rotation speed $\omega=5 \mathrm{rad} / \mathrm{s}$. We observe a gradual increase of the surface curvature with drum size. The upstream surface takes a parabolic shape, reminiscent of ballistic flight, extending to the center of the drum for the largest drum sizes. The downstream surface is nearly flat but develops a small crater as a result of the impact of ballistic particles for the largest drums. These features indicate that for $R / r \geq 100$, the flow is no longer in the cascading regime. Figure 11(b) shows the profiles for a constant Froude number $\mathrm{Fr}=0.8$ and different drum sizes. Here again, despite a constant Froude number, the profiles are different except for the largest drums where the profiles coincide. This suggests that the Froude number scales the profiles in the cataracting regime as the flow in the largest drums are in the cataracting regime.

To characterize the free surface shape, we consider two slope angles: 1) the secant slope $\theta_{m}$, and 2) the tangent slope $\theta_{\max }$ of the steepest descent along the free surface (see fig. 2). The angle $\theta_{m}$ represents the mean flow direction. Clearly, $\theta_{\max }$ reflects the kinematics of the free surface flow and the flow rate through the amount of feeding particles $\sim \omega$ whereas $\theta_{m}$ is a consequence of centrifugal forces $\sim \omega^{2}$ that determine the point from which the particles detach from the drum 


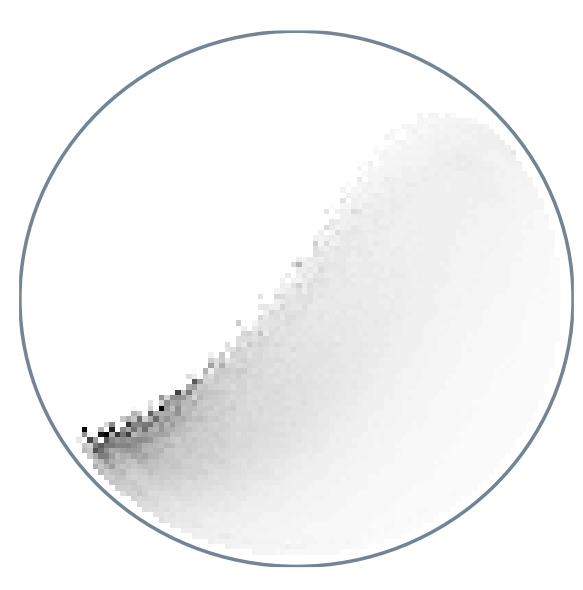

(a)

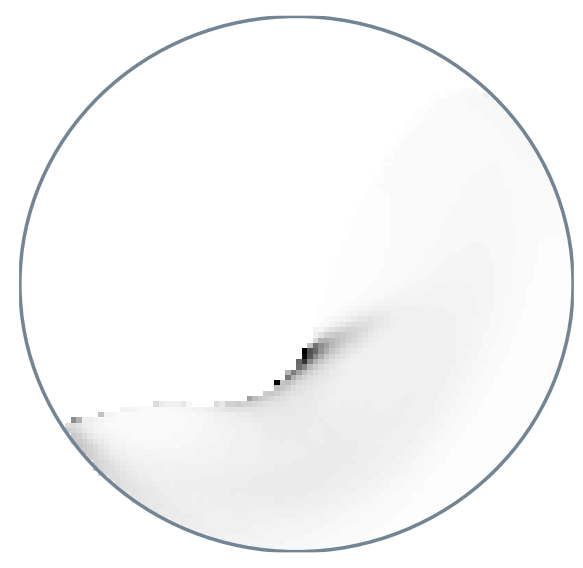

(c)

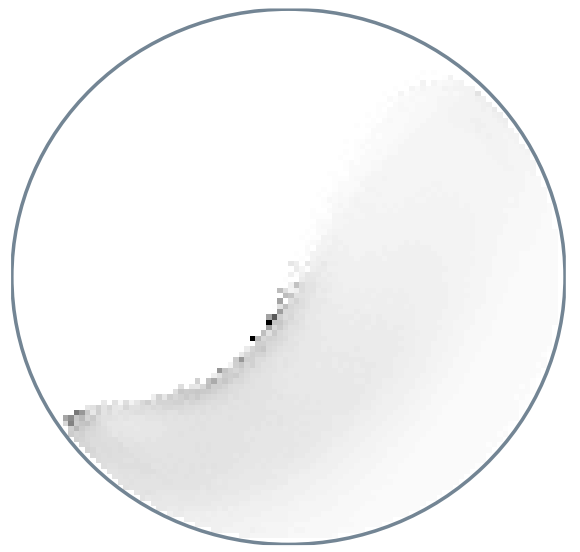

(b)

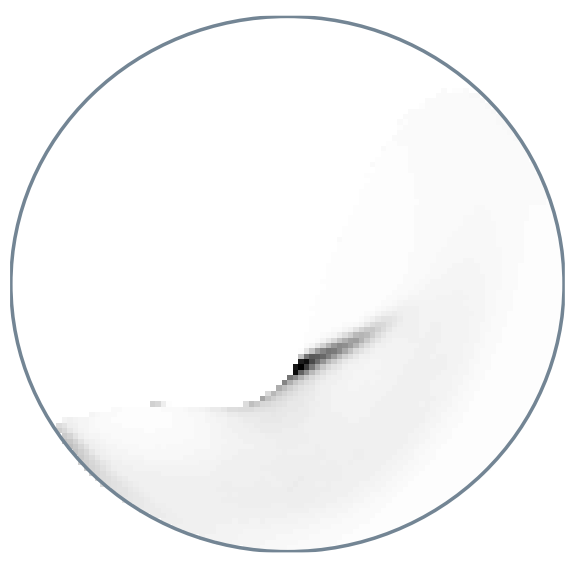

(d)

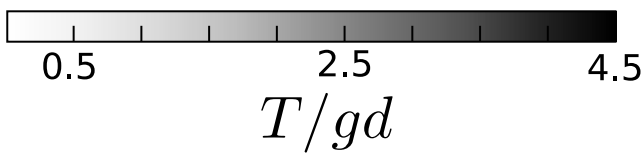

FIG. 10. Maps of granular temperature normalized by $g d$ in drums of four different size ratios $R / r$ : a) 18.75 , b) 37.5 , c) 93.75 and d) 125 , for $\mathrm{Fr}=0.8$.

wall. The ratio $\theta_{\max } / \theta_{m}$ is 1 in the rolling regime. It increases in the cascading regime and reaches values as high as 2 in transition to the cataracting regime.

Figure 12 shows $\theta_{m}, \theta_{\max }$ and their ratio as a function of $R / r$ for different values of parameters. The maximum angle $\theta_{\max }$ increases with $R / r$. The trends are similar but the initial values are different. They all end up at the same value $\theta_{\max } \simeq 1.25 \mathrm{rad}$ at large $R / r$. Again here, it should be noted that when Fr is kept constant, the increase of $R / r$ implies the decrease of $\omega$. The secant angle has a more dispersed aspect as a function of $R / r$. But if the last two data points for $\omega=$ $5 \mathrm{rad} / \mathrm{s}$, belonging to the cataracting regime, are ignored, we see that $\theta_{m}$ in all cases tends to 


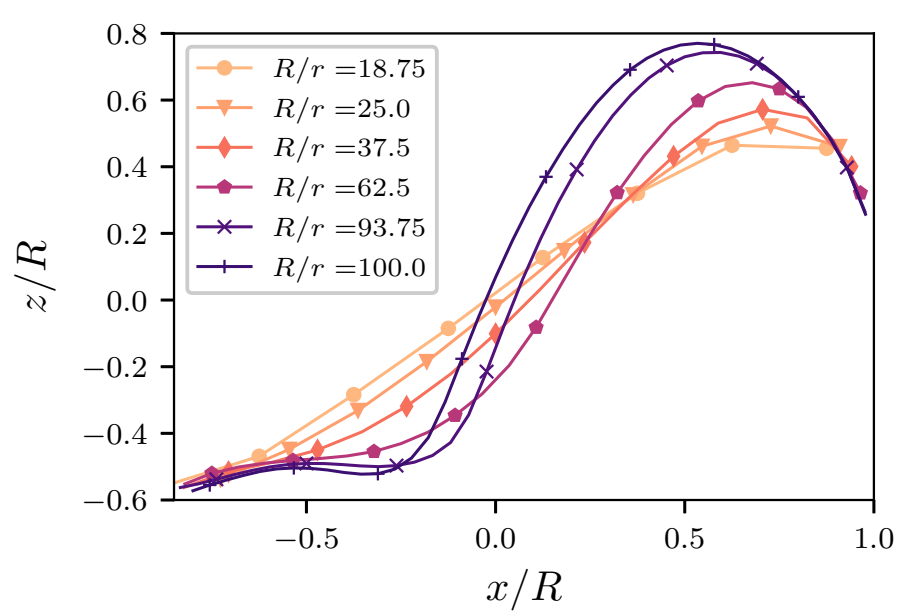

(a)

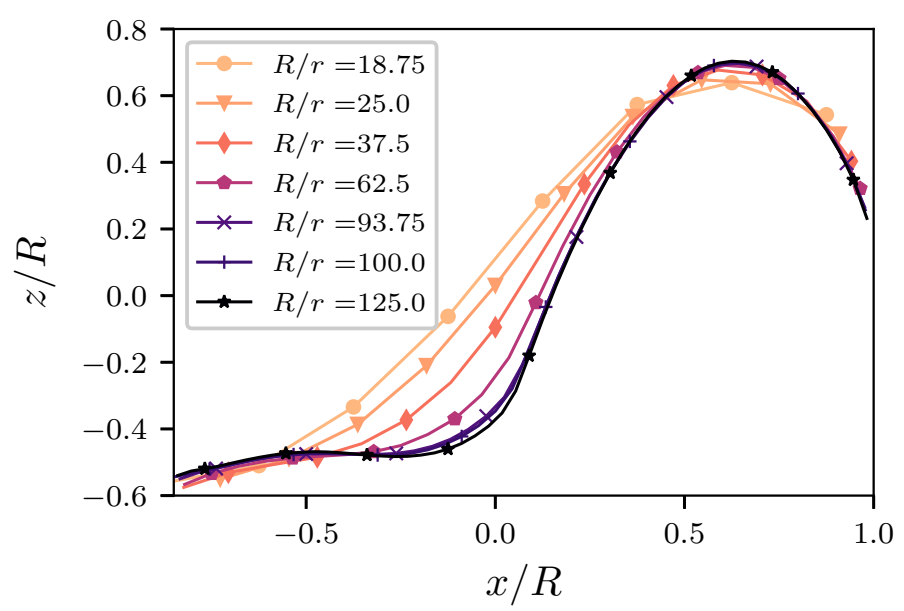

(b)

FIG. 11. Free surface profile for drums of different sizes at (a) $\omega$ constant and (b) Fr constant. The positions $x$ and $z$ are normalized by the drum radius $R$.

$0.62 \mathrm{rad}\left(\simeq 35^{\circ}\right)$ for large $R / r$. We see a more clear trend for $\theta_{\max } / \theta_{m}$, which is globally an increasing function of $R / r$ except for the two data points belonging to the cataracting regime. These observations clearly indicate that neither Fr nor $\omega$ can scale all the data. The scaling of the flow data will be discussed in section VI.

It is interesting to see how the surface profile as a kinematic property is correlated with the active layer thickness, which reflects the dynamics of the flow. Figure 13 shows $h_{a} / h_{b}$ as a function of both $\theta_{\max }$ and $\theta_{\max } / \theta_{m} . h_{a} / h_{b}$ grows with both $\theta_{\max }$ and $\theta_{\max } / \theta_{m}$ but the data points for different values of $\omega$ and $R$ gather much more tightly on a single curve for $\theta_{\max } / \theta_{m}$. This 

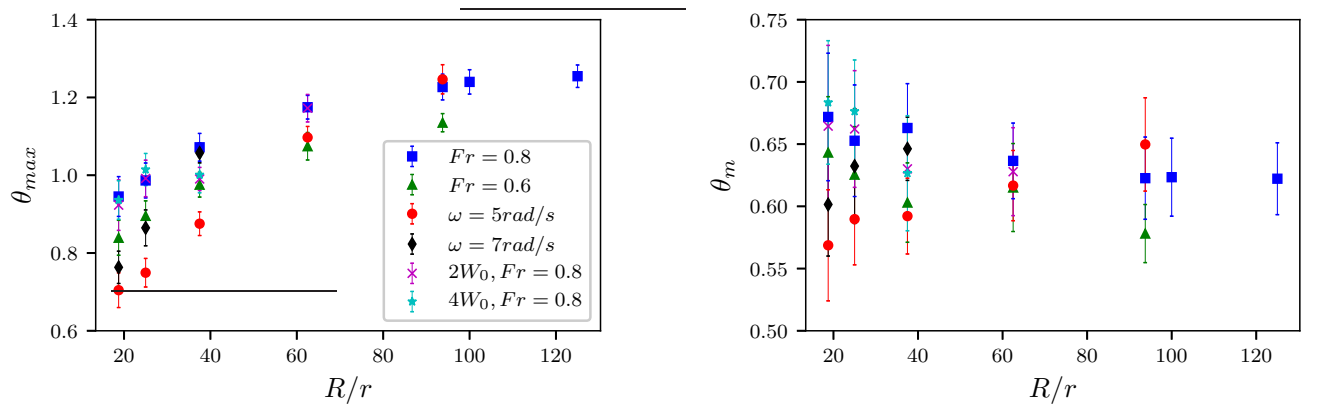

(a)

(b)

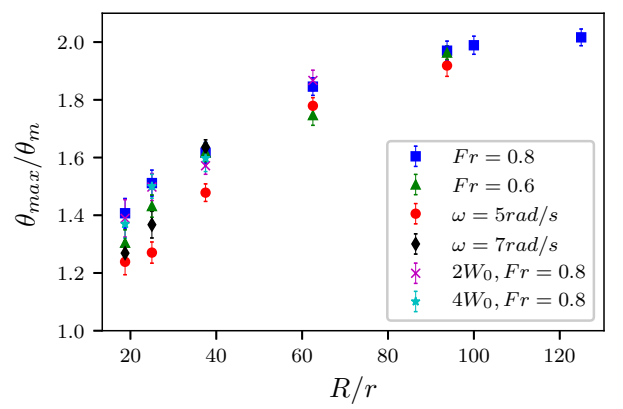

(c)

FIG. 12. Steepest descent angle $\theta_{\max }(\mathrm{a})$, secant angle $\theta_{m}$ (b) and the ratio $\theta_{\max } / \theta_{m}$ (c) as a function of $R / r$ for different parameter values. The error bars represent standard deviation of the values of angles.

correlation is nearly linear:

$$
\frac{h_{a}}{h_{b}}=0.31 \frac{\theta_{\max }}{\theta_{m}} .
$$

The lower limit of the cascading regime corresponds to $\theta_{\max } / \theta_{m}=1$, and by extrapolation of the linear fit we see that this point corresponds to $h_{a} / h_{b} \simeq 0.31$. Values of $h_{a} / h_{b}$ between 0.15 and 0.3 have been reported for rotating drums in the rolling regime ${ }^{42}$.

\section{FORCE DISTRIBUTIONS}

The particles flowing inside a rotating drum are subjected to the gravity forces in the vertical direction and centrifugal forces in radial directions. It is also well known that the contact forces are unevenly distributed inside granular materials and develop weak and strong force chains. Another important aspect in cascading flows is frequent collisions and inertial forces in the active surface layer. The combination of these features leads to complex force networks inside the drum as we observe on the snapshots of forces in drums of increasing size shown in Fig. 14. In smaller drums, we observe force chains encompassing the whole layer. These forces are often transient and they may occur in all parts of the flow. The presence of such long chains with correlation lengths close 


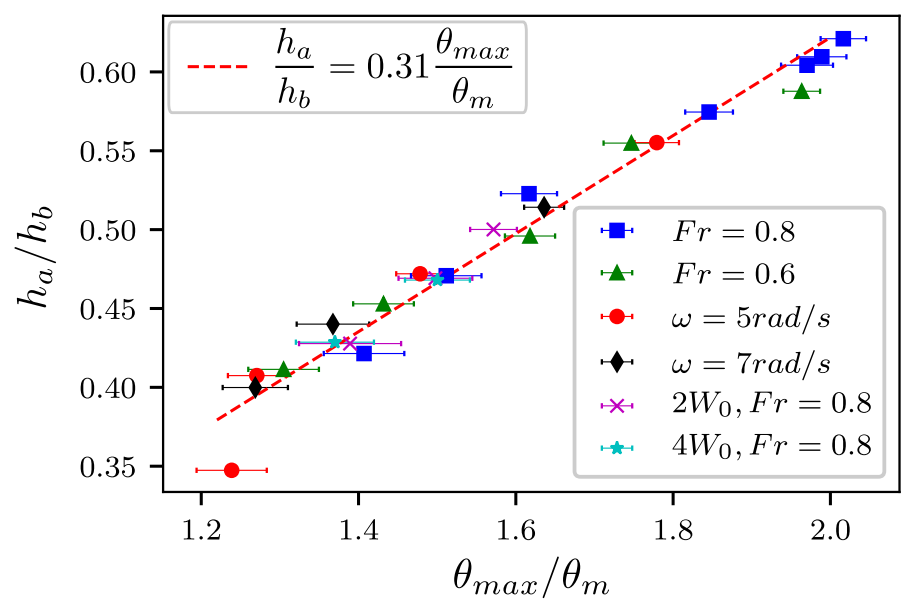

(a)

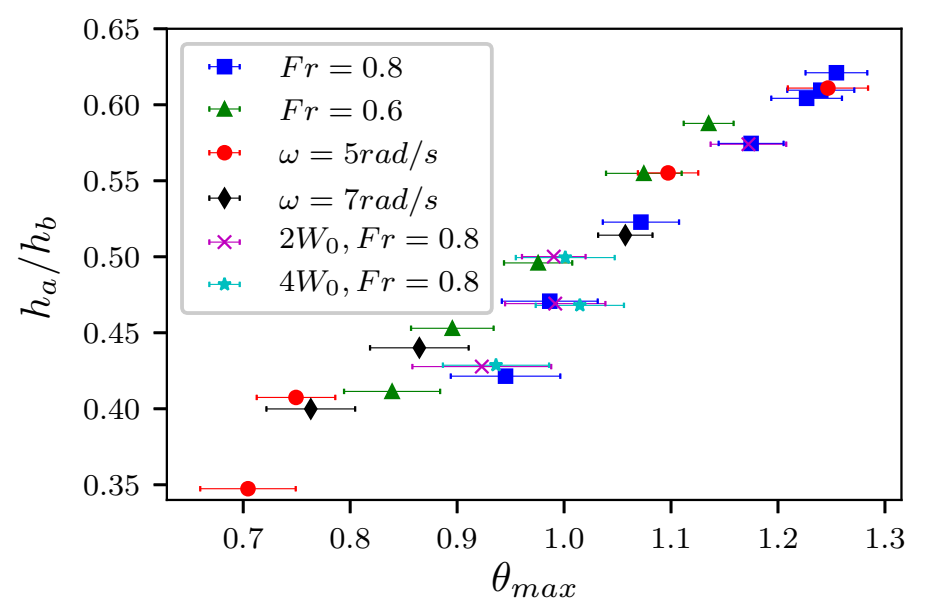

(b)

FIG. 13. a) Thickness $h_{a}$ of the active layer normalized by the bed thickness $h_{b}$ at the center of the drum as a function of $\theta_{\max } / \theta_{m}$ (a) and as a function of $\theta_{\max }(\mathrm{b})$, for simulations at either constant Fr or constant $\omega$. The error bars represent standard deviation of the values of $\theta_{\max }$.

to system size is at the origin of finite-size effects that we observe in small drums. For larger drums, the strong forces are much more diffuse and located at the center of the drum where the particles roll along the steepest descent of the free surface.

The probability density functions (pdf) of normal forces are shown on a log-linear scale in Fig. 15 for different values of drum size at constant Froude number Fr and constant rotation speed $\omega$. The distributions extend to very large forces $f_{n}$ compared to the mean force $\left\langle f_{n}\right\rangle$ and they fall off more slowly than an exponential function. With constant Froude number, they get broader with decreasing drum size (and hence increasing rotation speed) whereas with constant rotation speed 


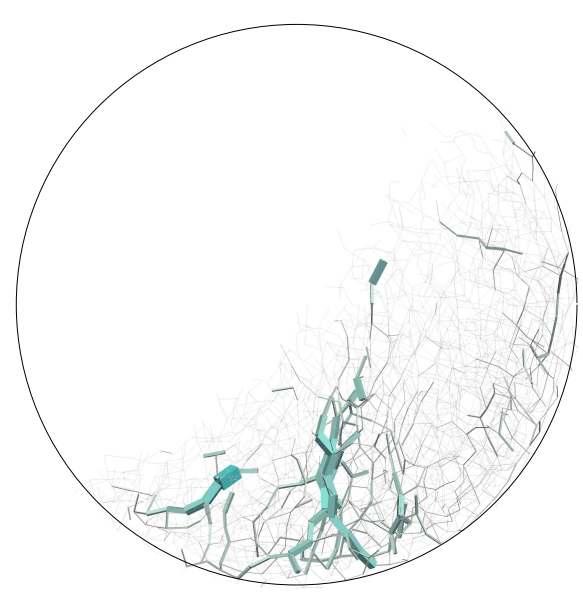

(a)

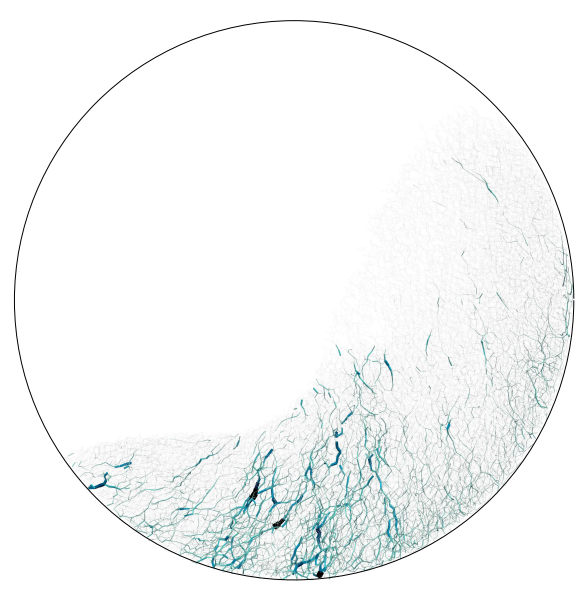

(c)

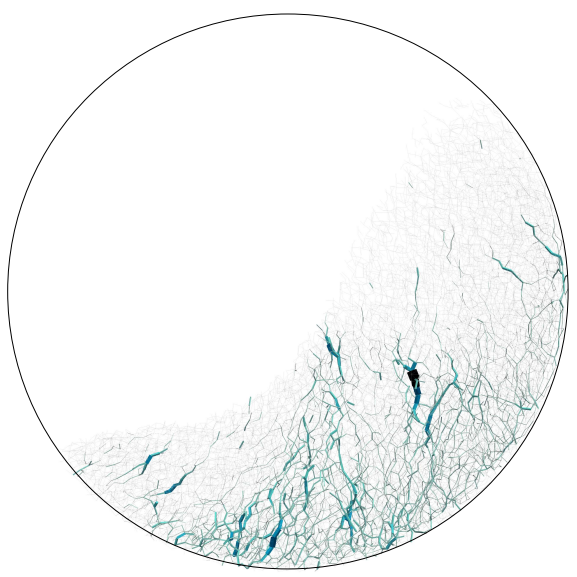

(b)

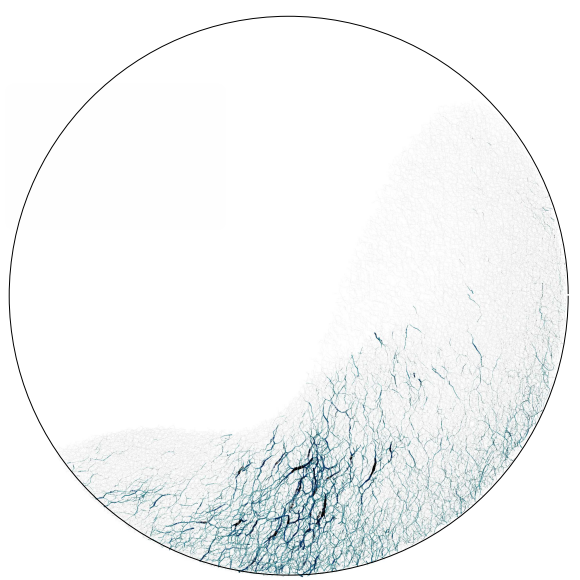

(d)

$$
\begin{aligned}
& \begin{array}{lllllll}
0.0 & 0.5 & 1 & 1.5 & 2 & 2.5 & 3.0
\end{array} \\
& f_{n} /\left\langle f_{n}\right\rangle
\end{aligned}
$$

FIG. 14. Contact force network inside drums of different sizes $R / r$ : a) 25, b) 62.5, c) 93.75, d) 125 . All drums have constant Froude number Fr=0.8. The thickness of the lines joining the particle centers is proportional to the corresponding normal force.

$\omega$ (and hence increasing Froude number) the forces change only slightly with drum size.

The nature of normal contact forces $f_{n}$ in a granular flow depends on the contact lifetimes. Short lifetimes are collisional and the force reflects the momentum exchange between two particles during a collision whereas long lifetimes belong to persistent contacts, which transmit external forces such as the particle weights. Although the collisions in dense granular flows are not 
binary and involves the whole contact network, it is possible to distinguish the impact forces by considering the forces only at new contacts formed between particles at each time step. Fig. 16 shows the pdf's of such impact forces normalized by the weight $m g R / r$ of a column of particles for increasing drum size with constant Froude number or constant rotation speed $\omega$. This normalization allows us to compare the orders of magnitude of impact forces with the static forces. We see that the impact forces can be far larger than the largest static forces (see orders of magnitude on the force axis in log scale). Remarkably, the data points nearly collapse in the case where $\omega$ is kept constant. This shows that the impact forces are proportional to $R / r$.

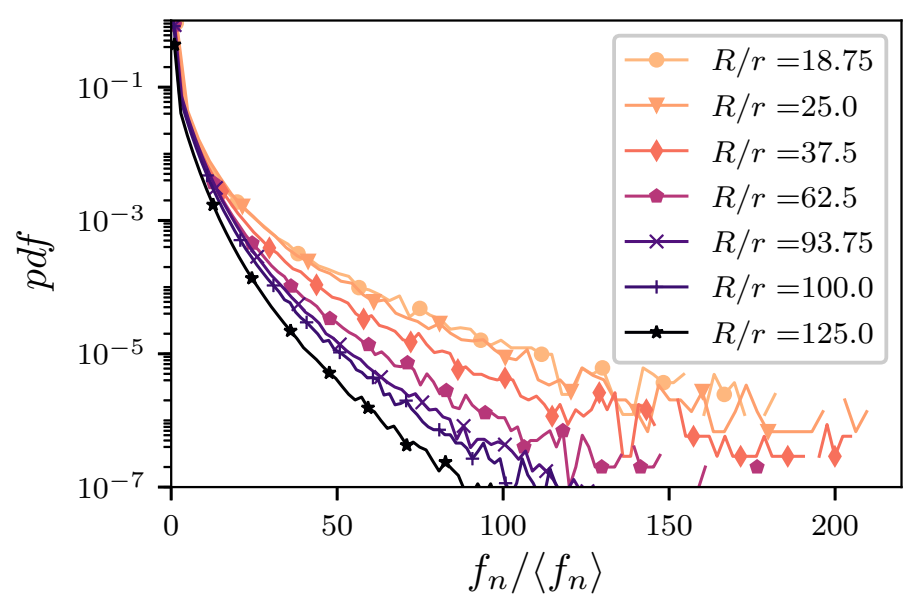

(a)

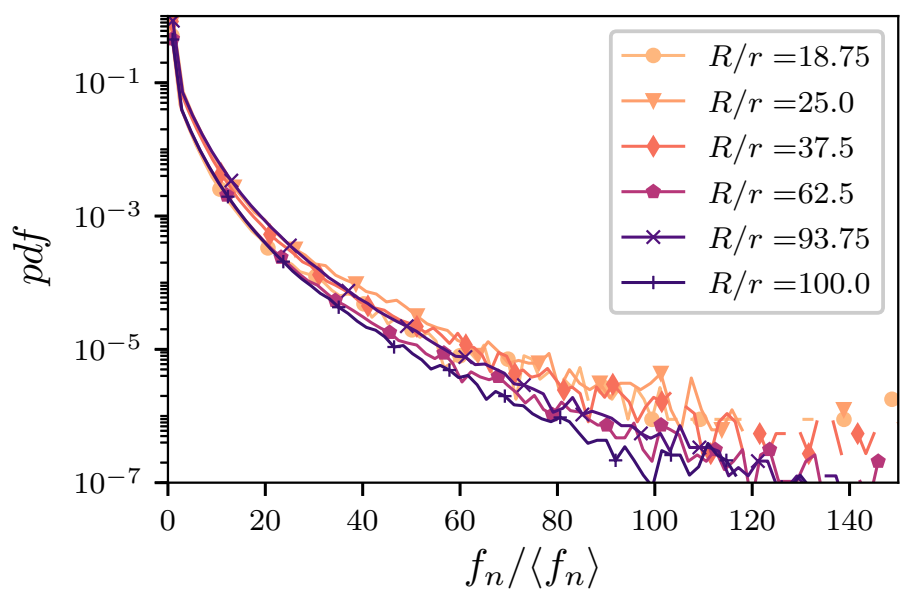

(b)

FIG. 15. Probability density function (pdf) of normal forces inside drums of different sizes $(R / r)$ at constant Froude number $\mathrm{Fr}=0.8$ (a) and at constant rotation speed $\omega=5 \mathrm{rad} / \mathrm{s}$ (b).

As the slope ratio $\theta_{\max } / \theta_{m}$ correlates well with the active layer thickness $h_{a}$ (Fig. 13), it is 


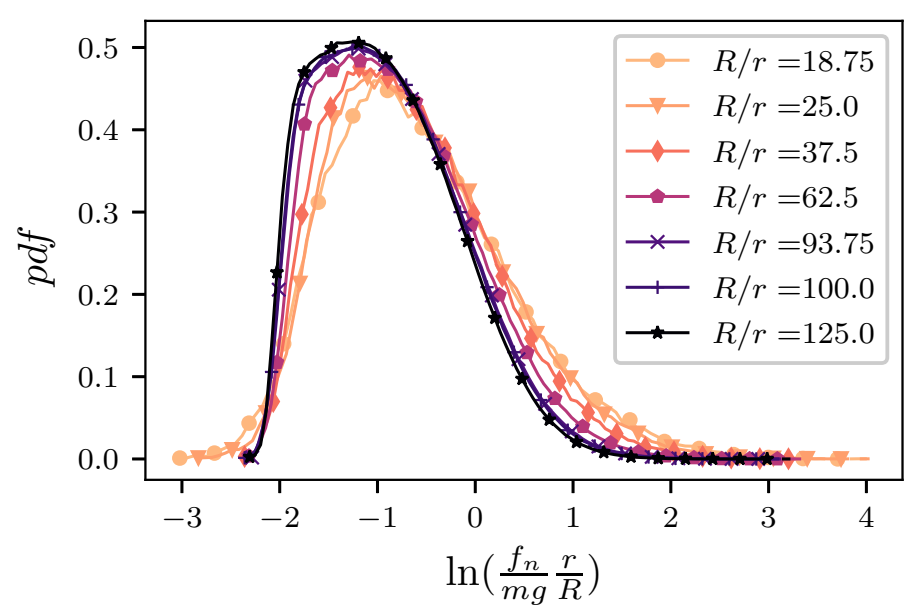

(a)

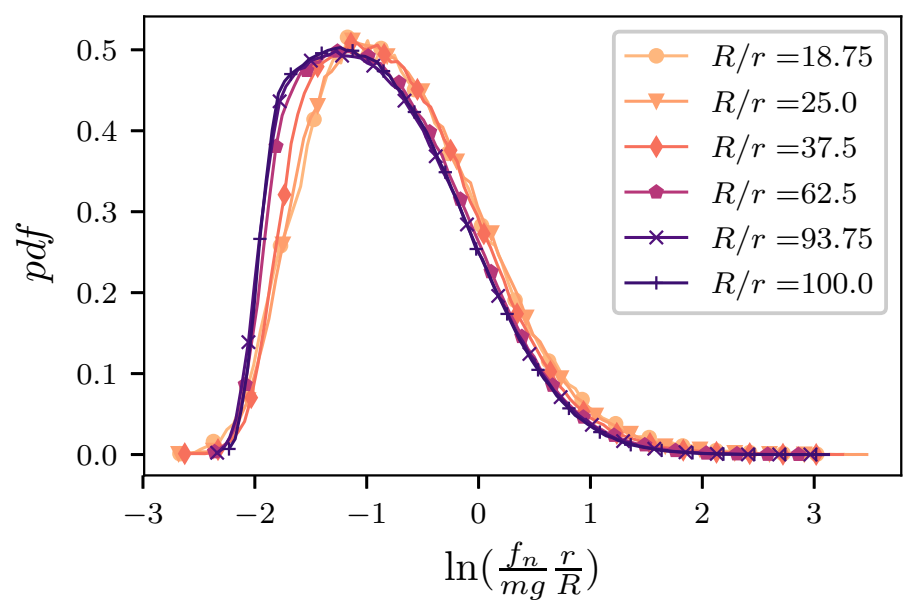

(b)

FIG. 16. Probability density function of the logarithm of normalized forces between colliding particles inside drums of different sizes $(R / r)$ at constant Froude number (a) and at constant rotation speed $\omega$ (b).

interesting to see whether the force distributions are also scaled by $\theta_{\max } / \theta_{m}$. The dynamic nature of the flow is directly related to force variability, which can be measured by the standard deviation $\Delta f=\left(\left\langle f^{2}\right\rangle-\langle f\rangle^{2}\right)^{1 / 2}$ of normalized forces, corresponding to the width of the force pdf's. Figure 17 shows $\Delta f$ as a function of $\theta_{\max } / \theta_{m}$ for all parameter values. We observe here a clear scaling that is well fit by a power-law

$$
\frac{\Delta f}{m g}=k\left(a-\theta_{\max } / \theta_{m}\right)^{n},
$$

with $k=16.08, a=2.5$ and $n=-1.18$. This scaling implies the divergence of $\Delta f$ as $\theta_{\max } / \theta_{m} \rightarrow$ 2.5, corresponding to transition from the cascading regime to the cataracting regime. This correlation between the force variability and surface profile is quite remarkable. It shows the 


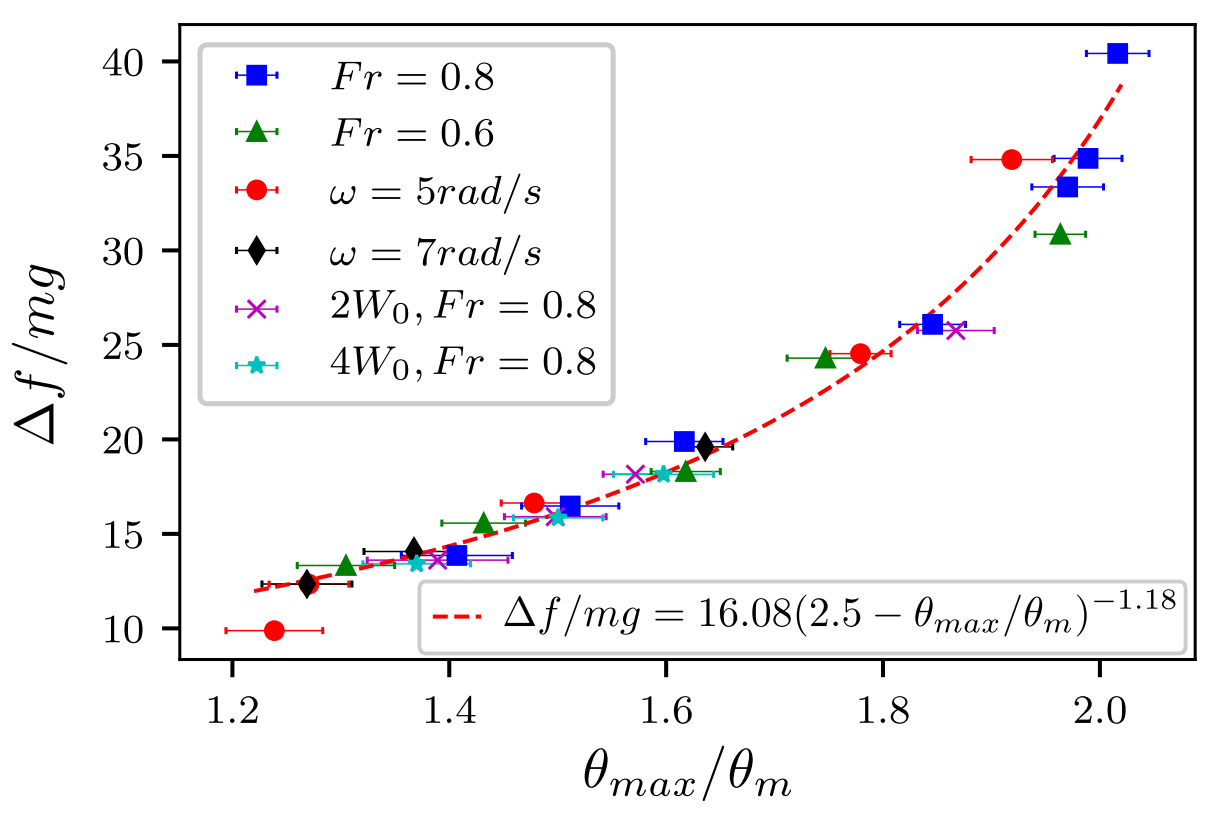

FIG. 17. Standard deviation $\Delta f$ of normal force pdf's as a function of $\theta_{\max } / \theta_{m}$ in drums of different sizes and values of system parameters. The dashed line is a power-law fit following equation 7 .

dynamic nature of the surface profile under the action of both centrifugal and inertial forces, and it suggests that the surface profile in the cascading regime may be used as upscaling factor when system parameters such as drum size and rotation speed change.

\section{SCALING OF CASCADING FLOWS}

In the previous sections, we investigated the influence of $\omega$ and $R$ on the velocity fields, free surface shape and contact forces in terms of various flow variables, including slope ratio $\theta_{\max } / \theta_{m}$, maximum slope $\theta_{\max }$, wall slip $S_{w}$, active layer thickness $h_{a}$ and force variability $\Delta f$ for a constant filling degree $J$. We showed that $\theta_{\max } / \theta_{m}, h_{a} / h_{b}$ and $\Delta f$ are related through power laws. This implies that $\theta_{\max } / \theta_{m}$ can be used as a scaling parameter for all flow properties. However, this analysis should be extended to define a scaling variable in terms of the operating parameters $\omega$ and $R$.

Our numerical results in the previous sections suggest that the flow behavior depends on both the Froude number, which accounts for the dynamic and inertial effects, and at least a second dimensionless parameter such as $R / d$ accounting for the geometric and finite size effects. We may 


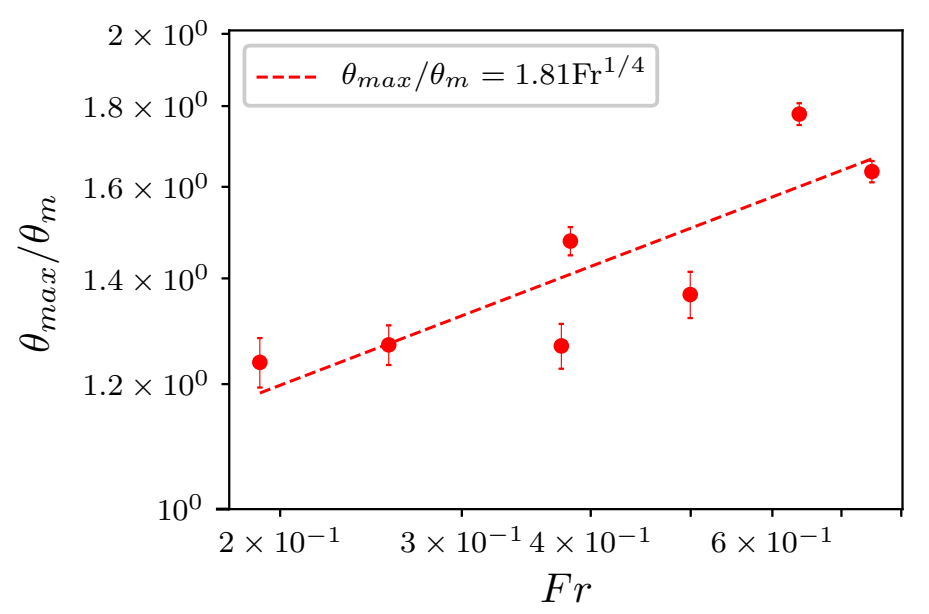

(a)

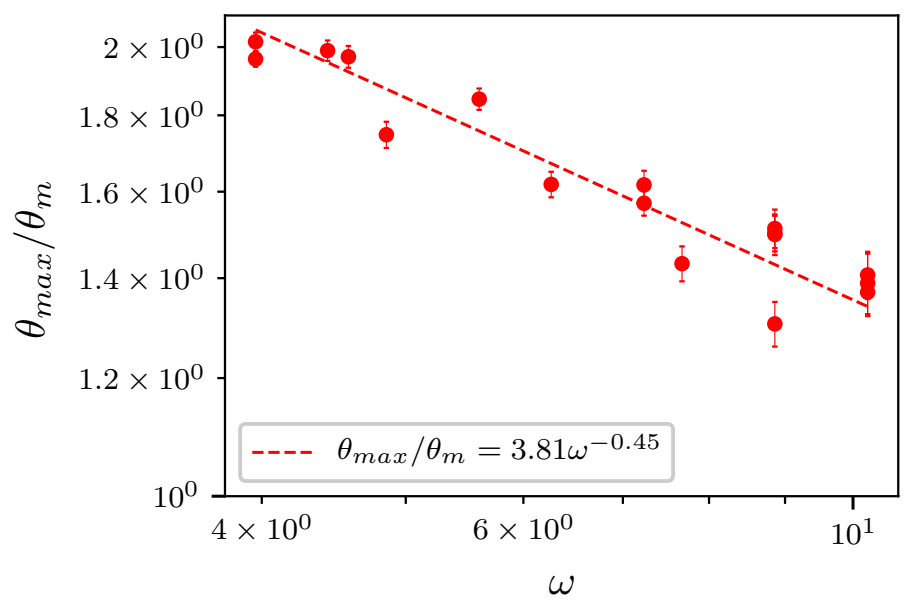

(b)

FIG. 18. Slope ratio $\theta_{\max } / \theta_{m}$ as a function of the Froude number Fr (a) and drum rotation speed $\omega$ (b) for all simulations. The dashed lines are power-law trends with exponents $1 / 4$ and -0.45 , respectively. The error bars represent standard deviation of the values of $\theta_{\max }$.

also consider the filling ratio $J$, which is a dimensionless parameter characterizing the geometry of the flow. In the absence of a full physically justified parameter, a simple way to define a general dimensionless scale parameter consists in searching for a parameter $\Upsilon$ of the following general form:

$$
\Upsilon=\left(\frac{R \omega^{2}}{g}\right)^{\alpha}\left(\frac{R}{d}\right)^{\beta} J^{\gamma}
$$

where the exponents $\alpha, \beta$ and $\gamma$ will be fixed from the simulation data. This scaling parameter is proportional to $\omega^{2 \alpha} R^{\alpha+\beta-\gamma}$ since $J=h_{0} / 2 R$. As the variables $\theta_{\max } / \theta_{m}, h_{a} / h_{b}$ and $\Delta f$ are correlated, we may use any of them to get the best collapse of the data as a function of $\Upsilon$. We 


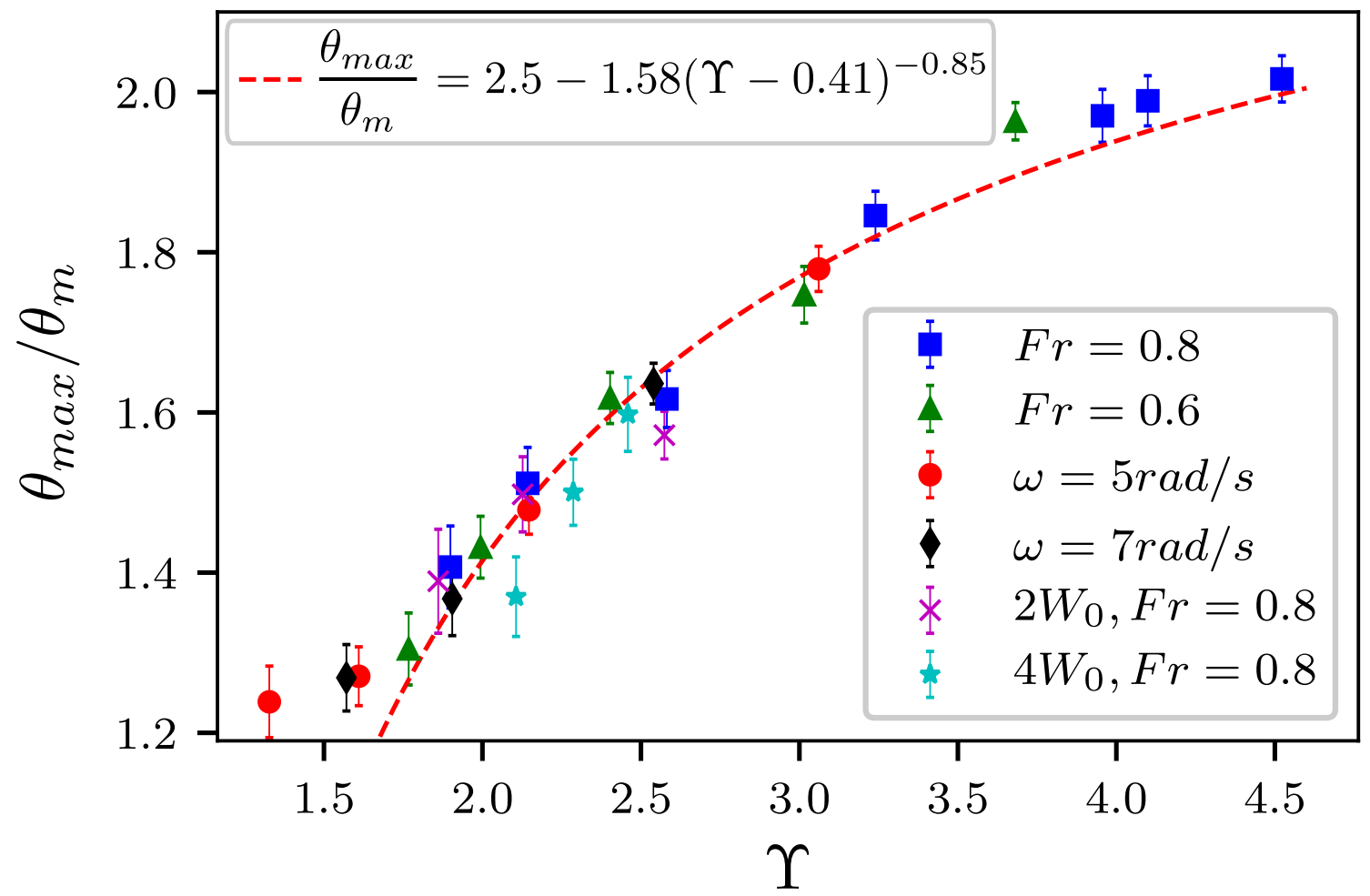

FIG. 19. Slope ratio $\theta_{\max } / \theta_{m}$ as a function scaling parameter $\Upsilon$ defined by equation (8) with $\alpha=1 / 4$ and $\beta=1 / 2$. The dashed line is the fitting form given by equation (9). The error bars represent standard deviation of the values of $\theta_{\max }$.

used $\theta_{\max } / \theta_{m}$ for this purpose, and in order to obtain a first estimate of the values of $\alpha$ and $\beta$, we plotted $\theta_{\max } / \theta_{m}$ separately as a function of Fr and $\omega$ for all our simulations with $J=0.45$, as shown in Fig. 18. The observed trends can be modeled as power laws with exponents $\sim 1 / 4$ and $\sim-0.45$, respectively. From these exponents, we get $\alpha \simeq 1 / 4$ and $\beta \simeq 1 / 2$.

Since we consider here the behavior for a constant value $J=0.45$ of the filling degree, we plot in Fig. 19 the values of $\theta_{\max } / \theta_{m}$ as a function of $\Upsilon$ by setting $J^{\gamma} \simeq 1.22$. At this point, this value is a simple numerical factor that does not affect the scaling behavior. We could also set its value to 1, but we shall see below the reason/justification behind the above choice. Figure 19 shows that $\Upsilon$ with $\alpha=1 / 4$ and $\beta=1 / 2$ leads to a good collapse of the data on a master curve. It is well fitted by

$$
\frac{\theta_{\max }}{\theta_{m}}=a-b(\Upsilon-0.41)^{m}
$$




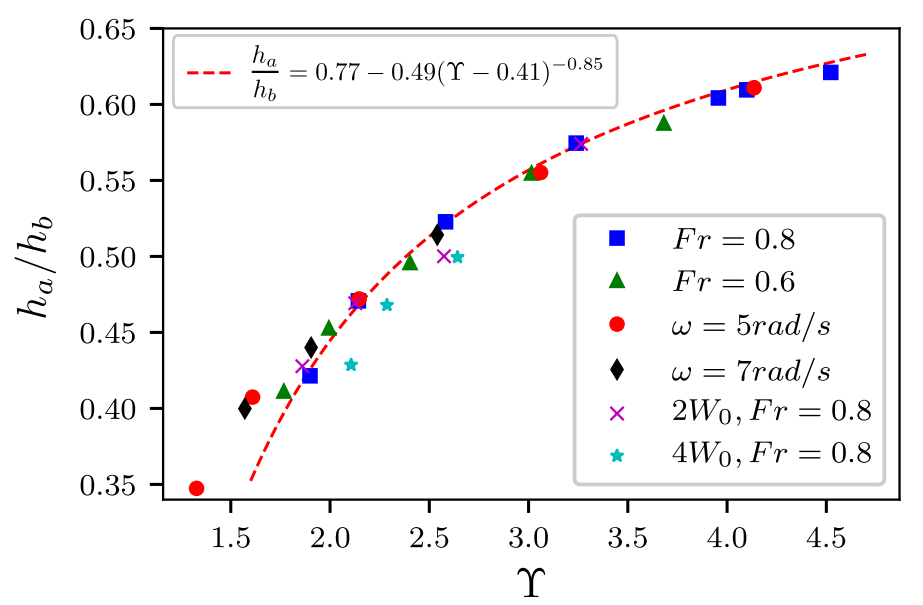

(a)

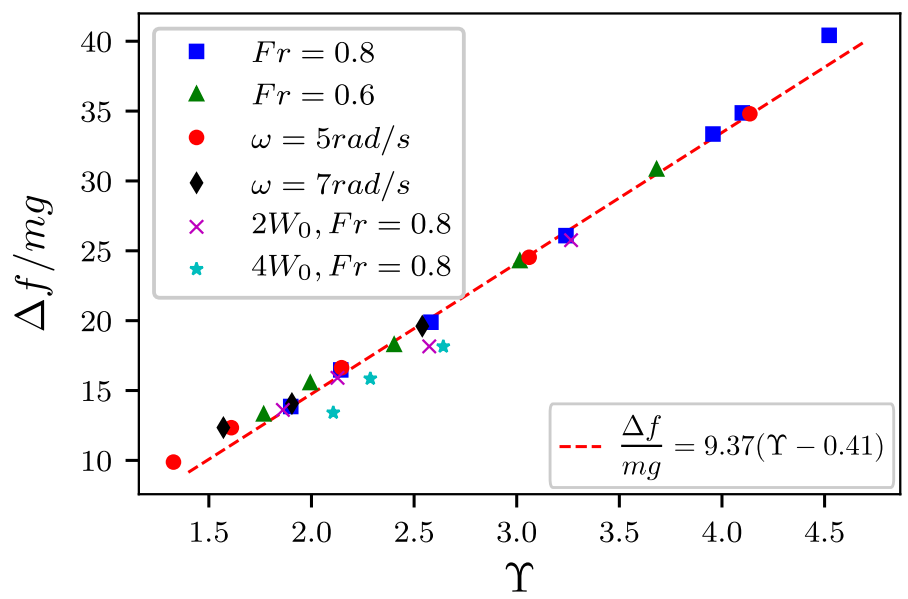

(b)

FIG. 20. Normalized active layer thickness (a) and standard deviation of the normal force distribution (b) as a function of the scaling parameter $\Upsilon$ defined by (8) with $\alpha=1 / 4$ and $\beta=1 / 2$.

with $b=1.58$ and $m=-0.85$. Only the data points at small values of $\Upsilon$ (corresponding to small values of $R / d$ ) are slightly off the fit. These points represent also small values of $\theta_{\max } / \theta_{m}(\simeq 1.2)$ and belong thus to the transition state from the rolling regime to the cascading regime. In this state, the centrifugal forces are small and the flow is mainly governed by inertial forces. The lowest value of $\theta_{\max } / \theta_{m}$ is 1 and, according to equation (9) is reached for $\Upsilon \simeq 1.47$. On the other hand, as in equation (7), $\theta_{\max } / \theta_{m}$ tends to $a=2.5$ when $\Upsilon$ increases. Hence, $\Upsilon$ varies in the range $[1.47 ; 4.5]$, which can be considered as the values of $\Upsilon$ that characterize the cascading regime independently of the specific values of $\omega, R, d$ and $g$.

In a similar vein, we expect other flow variables to scale with $\Upsilon$. This is what we see in Fig. 


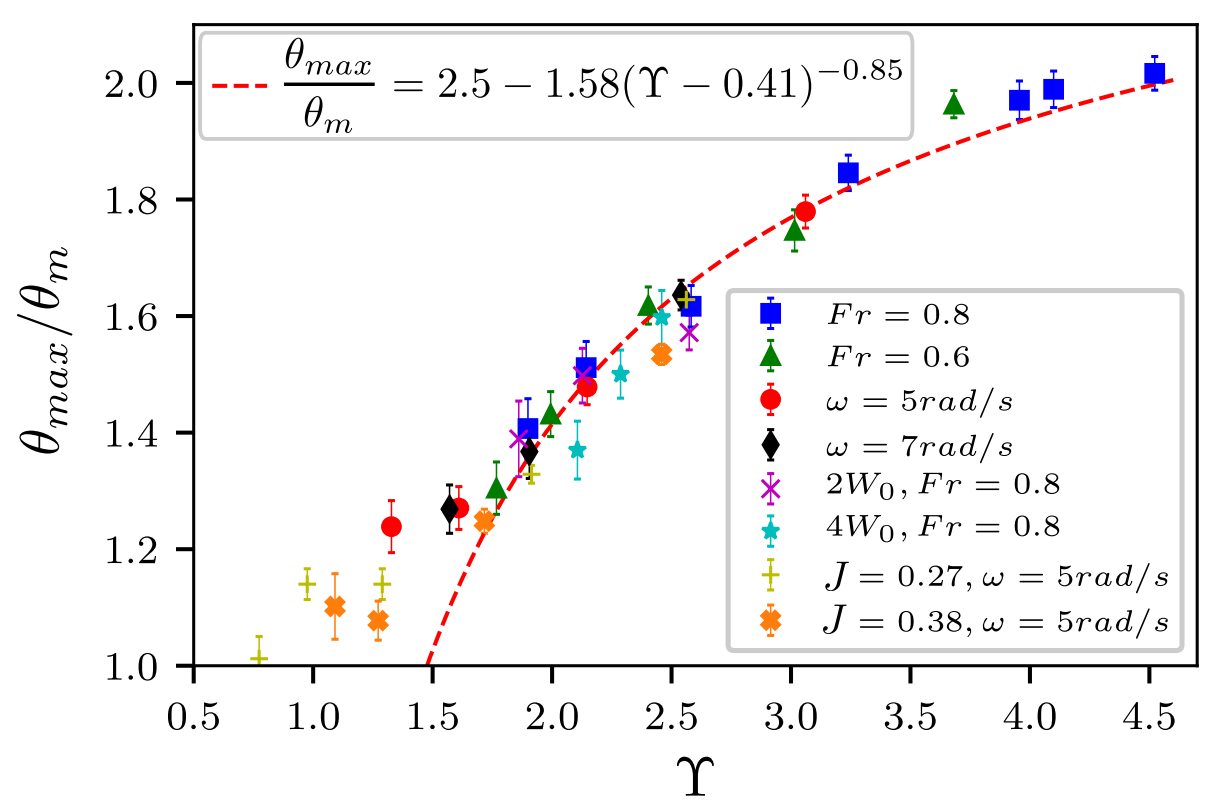

FIG. 21. Slope ratio as a function of the scaling parameter $\Upsilon$ defined by equation (8) with a range of values of drum size $R$, rotation speed $\omega$ and filling degree $J$ with $\alpha=1 / 4, \beta=1 / 2, \gamma=1$. The dashed line is a power-law fitting form. The error bars represent standard deviation of the values of $\theta_{\max }$.

20, showing the normalized active layer thickness $h_{a} / h_{b}$ and force variability $\Delta f$ as a function of $\Upsilon$. The fitting forms are

$$
\begin{aligned}
\frac{h_{a}}{h_{b}} & =c-e(\Upsilon-0.41)^{m}, \\
\frac{\Delta f}{m g} & =h(\Upsilon-0.41),
\end{aligned}
$$

with $c=0.77, e=0.49$ and $h=9.37$. The offset value 0.41 of $\Upsilon$ was adopted from equation (9). Note that, from equations (7), (10), (11) and (9), we have $m n=1$ and $h=k b^{n}$. The linear relation between $\Delta f$ and $\Upsilon$ is quite remarkable and unexpected. As force fluctuations play a major role in the breakage and agglomeration processes, this linear scaling predicts that $\Upsilon$ can also be the relevant scaling parameter for such processes.

The scaling parameter $\Upsilon$ in all the data previously analyzed does not take into account the filling degree $J=h_{0} / 2 R$, which was fixed to 0.45 . We performed a series of simulations with two lower values $J=0.38$ and $J=0.27$ of the filling degree, two different values of $\omega$ and several values of $R / d$. In order to determine the value of the exponent $\gamma$ in (8), we plotted all simulated values of slope ratio for all values of $J$ as a function of $\Upsilon$ with different values of the exponent $\gamma$. We find a 
reasonable collapse of all the data for $\gamma=1$, as shown in Fig. 21. Equation (9) fits well the data up to the observed fluctuations for lower values of $J$. They correspond to small values of $\Upsilon$ and slope ratio in transition from rolling to cascading regime. It is important to note that this scaling holds for filling degrees $J$ below 0.5 . While we did not check the scaling for higher values, we believe that flow regimes are different for $J>0.5$ due to the increasingly smaller available volume for the development of surface flow.

The scaling parameter $\Upsilon$ with $\alpha=1 / 4, \beta=1 / 2$ and $\gamma=1$ may be recast in different forms. For example, it can be rewritten as

$$
\Upsilon=\left\{\omega\left(\frac{R}{g}\right)^{1 / 2} \frac{h_{0}}{2 R} \frac{h_{0}}{d}\right\}^{1 / 2} .
$$

This form highlights two size factors $h_{0} / d$ and $J=h_{0} / 2 R$, representing the finite size effect and the filling degree, respectively. The dimensionless factor $\omega \sqrt{R / g}=\operatorname{Fr}^{1 / 2}$ is simply the ratio of free-fall time (over a distance of the order of drum size) $\sqrt{R / g}$ to the imposed time $\omega^{-1}$ by drum rotation. With increasing free-fall time, the upstream particles, whose flux is proportional to $\omega R$, accumulate and form an increasingly curved surface, which, by increasing the free surface length, 'adapts' the effective cascading time to the imposed feeding time by drum rotation. The transition to the cataracting regime takes place when this ratio is close to 1 .

In all our simulations, we kept a constant particle size $d=8 \mathrm{~mm}$. The point is that the frictional contacts are the only interactions between particles, and they do not involve the size of the particles. This implies that multiplying all lengths and the gravity $g$ by the same factor should not change the flow behavior. This symmetry is included in the scale parameter $\Upsilon$ through the ratios $R / d$ and $R \Omega^{2} / g$. For this reason, the same scaling is expected to hold when $d$ is changed. It is also obvious that if $\Upsilon$ is the scale parameter, any power of $\Upsilon$ can be used as scale parameter. In particular, we may use $\Upsilon^{2}$ to remove the exponent $1 / 2$ in equation (12). However, the range of values of higher-order parameters like $\Upsilon^{t}$ increases considerably with the power $t$ (up to 100 with $t=2$ ), tending to mask the fluctuations of flow variables (reflecting finite size effects) at small values of $\Upsilon$.

Similar scaling parameters have been proposed by other authors. Taberlet et al. ${ }^{15}$ proposed the parameter

$$
\Lambda=\left(\operatorname{Fr} \frac{d}{R}\right)^{1 / 4} \frac{R}{W},
$$

which involves $\mathrm{Fr}, R / d$ and $R / W$. This parameter was deduced from a theoretical analysis of the 


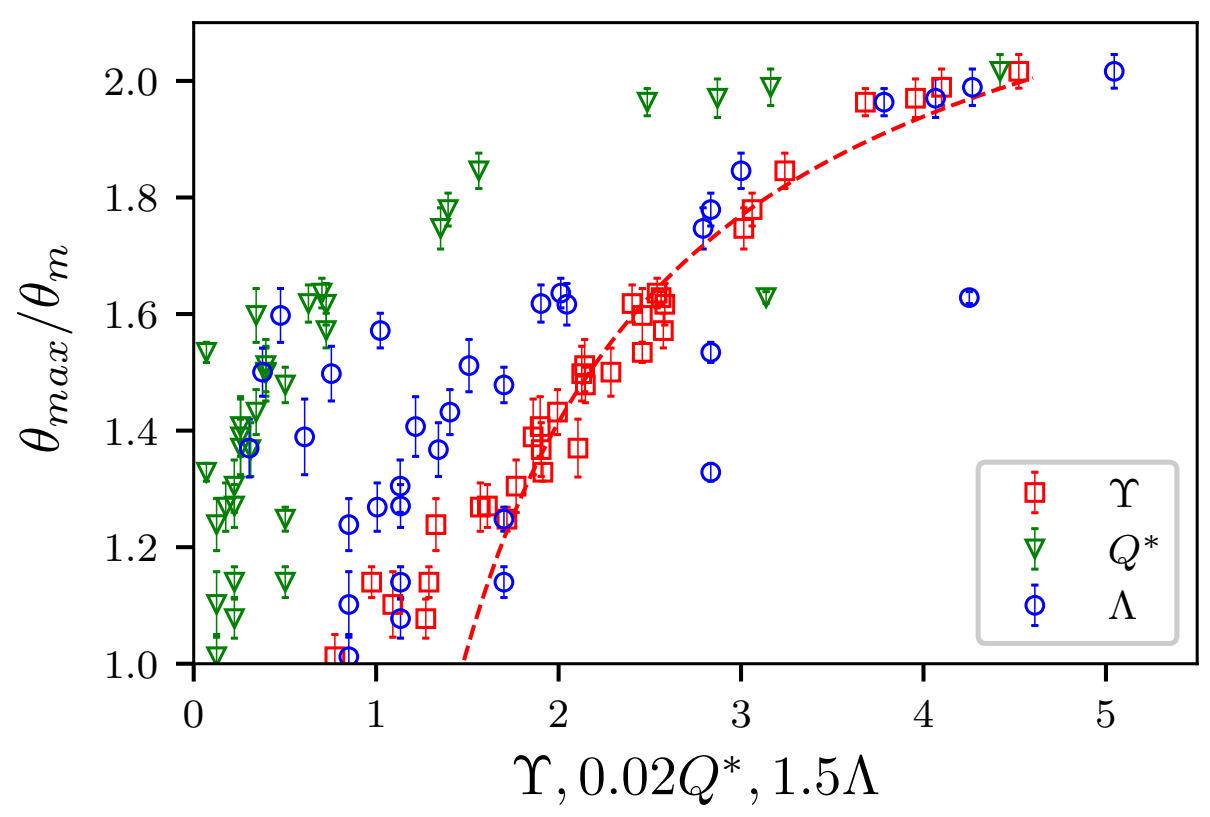

FIG. 22. Slope ratio as a function of $\Upsilon, Q^{*}$ and $\Lambda$; see text. For the sake of comparison between the curves in the same range of values as $\Upsilon, Q^{*}$ and $\Lambda$ are multiplied by prefactors. The dashed line follows equation 9. The error bars represent standard deviation of the values of $\theta_{\max }$.

S-shape of the free surface and its relation with the length of the drum and/or friction with the end walls. Pignatel et al. ${ }^{20}$ introduced the parameter

$$
Q^{*}=\frac{1}{2} \operatorname{Fr}^{1 / 2}\left(\frac{R}{d}\right)^{3 / 2},
$$

which was used for the scaling of the flowing layer thickness. Both these scalings involve rotating drums with lateral walls.

In Fig. 22 we have plotted $\theta_{\max } / \theta_{m}$ as a function of $\Upsilon, \Lambda$ and $Q^{*}$ by multiplying $\Lambda$ and $Q^{*}$ by a scale factor in order to bring all the points to the range of values of $\Upsilon$. We see that most data points as a function of $\Lambda$ and $Q^{*}$ collapse and show a trend that is similar to the same data as a function $\Upsilon$. In both cases, a few points are off the collapsed part of the curve. Clearly, these scalings cannot be strictly compared with $\Upsilon$ as they miss the variation of the filling degree parameter and it is not clear whether they can be considered to be in the rolling or cascading regime. In particular, in the work by Taberlet et al., the shape of the free surface changes as a function of the drum length or friction with the end walls whereas in our case the free surface shape changes due to the effect of drum size on the wall slip. This suggests that the drum length and/or friction with the end walls 
affect the surface shape through their effect on wall slip. Further investigation in this direction can be useful for a better understanding of wall effects.

\section{DISCUSSION AND CONCLUSIONS}

In this paper, we investigated the rheology of granular materials in rotating drums in the cascading regime by means of numerical simulations. We introduced a scaling parameter that comprises various system parameters and consistently describes flow variables such as surface flow thickness, wall slip, free surface curvature and contact force variability. The cascading flow regime is characterized by combined effects of centrifugal and inertial forces that induce a curved free surface and rapid flow along its steepest descent. A transition to the cataracting regime occurs when the centrifugal force prevails and the particles at the free surface are no more bound together by frictional contact in a dense flow, but tend to follow ballistic motions.

The simulations revealed well-defined relations between slope ratio, flow thickness, wall slip and force variability. In particular, the wall slip is larger in smaller drums, implying a lower particle feed to the free surface and thus, a less curved free surface for the same values of the Froude number and rotation speed. The force variability increases with slope ratio and flow thickness. With periodic boundary conditions, the drum width is not a relevant parameter and there is no flow in the lateral directions. The wall slip can thus be considered as the main effect of drum size as compared to particle size, with dramatic consequences for the free surface profile and active layer thickness. The wall slip needs a more detailed analysis for a better understanding of its nature. In our simulations, the wall-particle coupling is purely frictional. Wall roughness may alter the coupling, but its effect may be just equivalent to a higher friction coefficient. It is also well known that high friction coefficient or wall roughness leads to slip between granular layers close to the wall so that the effective friction coefficient with the wall will be that of particles ${ }^{46}$. Hence, the wall slip should not be confused with simple sliding of the particles against the drum wall as in frictional slip between two solid bodies. It has rather a collective nature in the sense of higher shearing at the walls as compared to the bulk. Then, it should be treated with the same status as other flow variables. This is the picture suggested by our simulations with well-defined relationships between wall slip, active flow thickness and force fluctuations although it does not elude a more close look into its nature by direct comparison with simulations with rough walls. It can also be relevant to consider particles of aspherical shapes such as polyhedral particles. The 
effect of particle shape on wall slip can be similar to wall roughness.

The observed relation between force variability and free surface profile is quite remarkable. We have seen that, as a result of collisions and inertial dynamics, the contact forces are frequently well above the mean particle weight. Our simulations indicate that high shear stresses are located below the steepest descent, providing thus a plausible reason for such a strong correlation between forces and slope ratio. The strong force chains and impacts are responsible for particle breakage in the milling process. They are also important in the granulation process of wet particles that involves the capture and erosion of wet agglomerates during granule growth as a result of interactions with the surrounding particles. It can thus be conjectured that surface profiles, in correlation with force fluctuations inside a rotating drum, can be measured and used as a scale-up parameter in the milling and granulation processes.

The scaling parameter $\Upsilon=\operatorname{Fr}^{1 / 4}(R / d)^{1 / 2} J$ introduced in this work from drum size $R$, rotation speed $\omega$, filling degree $J$, and particle size $d$, is consistent with all our simulation data and a scaling previously introduced by another author ${ }^{20}$. Up to a scaling factor $h_{0} / d$, this dimensionless parameter can be seen as the ratio between the free-fall time and the feeding time imposed by drum rotation. In the steady state, the effective cascading time is longer than the free-fall time due to the free surface curvature. Hence, the potential energy of the particles fed into the flow is not only dissipated by frictional contacts, but also partially transforms into kinetic energy that is dissipated later by pushing the particles to a higher position in the upstream heap.

This mechanism is different from that in the rolling and cataracting regimes. In the rolling regime, the kinetic energy induced by drum rotation is not significant, and the role of drum rotation is simply to increase the overall potential energy of the particles by bringing them up to the upstream surface tip. Hence, the rolling regime is mainly governed by inertial flow at the flat free surface with a thickness and inclination depending on the rotation speed. This is the reason why this configuration is well suited to the investigation of uniform inertial flows. On the other hand, in the cataracting regime, the dense inertial free surface flow is replaced by a fluidized bed and ballistic particle motions with an increasing altitude of ejected particles for larger values of the Froude number. The parameter $\Upsilon$ scales well the slope ratio and force variability and may be used to upscale drum size from laboratory to industrial scale. This scaling works down to small ratios $R / d$ or $h_{0} / d$ where finite size effects seem to prevail and the surface is close to the rolling regime even for high values of rotation speed.

This work can be extended to drums of larger width and even larger sizes than those considered 
in this paper. The width of the drum in our simulations had no significant effect on the flow due to periodic lateral boundaries. For this reason, we do not expect it to play a role for larger drums. It is therefore more interesting to remove periodic boundaries in the future work and perform a similar analysis in order to get a better understanding of the finite size effects arising from the ratio $W / d$ or from the aspect ratio $R / W$. Another parameter that can be relevant to the cascading regime and the values of fitting parameters is the restitution coefficient. It was set to zero in all our simulations due to the dense nature of the flow, but it may affect the transition to the cataracting regime. In the same way, the finite size effects need to be investigated by considering larger ratios of drum size and filling height to the particle size. Finally, an interesting direction of research is the application of the scaling proposed in this paper to the grinding process in a rotating drum with crushable particles, where the evolution of particle breakage may be correlated with $\Upsilon$ and slope ratio.

\section{REFERENCES}

${ }^{1}$ M. Cacciuttolo and A. Arunakumari, Scale-Up Considerations for Biotechnology-Derived Products (CRC Press, 2005) pp. 129-160.

${ }^{2}$ L. M. Tavares, KONA Powder and Particle Journal 2017, 106 (2017).

${ }^{3}$ A. Brunaugh and H. D. Smyth, Drug Delivery and Translational Research 8, 1740 (2018).

${ }^{4}$ T. T. Vo, S. Nezamabadi, P. Mutabaruka, J.-Y. Delenne, E. Izard, R. Pellenq, and F. Radjai, The European Physical Journal E 42, 127 (2019).

${ }^{5}$ J. Rajchenbach, Physical Review Letters (1990), 10.1103/PhysRevLett.65.2221.

${ }^{6}$ S. Longo and A. Lamberti, Experiments in Fluids 32, 313 (2002).

${ }^{7}$ A. V. Orpe and D. V. Khakhar, Journal of Fluid Mechanics 571, 1 (2007).

${ }^{8}$ D. Bonamy, P. H. Chavanis, P. P. Cortet, F. Daviaud, B. Dubrulle, and M. Renouf, European Physical Journal B 68, 619 (2009).

${ }^{9}$ I. Govender, M. C. Richter, A. N. Mainza, and D. N. De Klerk, AIChE Journal 63, 903 (2017).

${ }^{10}$ É. Azéma, F. Radjai, and J. N. Roux, European Physical Journal E 41 (2018), 10.1140/epje/i2018-11608-9.

${ }^{11}$ D. Cantor, E. Azéma, P. Sornay, and F. Radjai, Physical Review E 98, 1 (2018).

${ }^{12}$ A. V. Orpe and D. V. Khakhar, Physical review. E 64 (2001), 10.1103/PhysRevE.64.031302.

${ }^{13}$ Y. L. Ding, R. Forster, J. P. K. Seville, and D. J. Parker, Powder Technology 124, 18 (2002).

${ }^{14}$ A. Alexander, T. Shinbrot, and F. J. Muzzio, Powder Technology 126, 174 (2002). 
${ }^{15}$ N. Taberlet, P. Richard, and E. John Hinch, Physical Review E - Statistical, Nonlinear, and Soft Matter Physics 0607085 .

${ }^{16}$ T. Iwasaki, T. Yabuuchi, H. Nakagawa, and S. Watano,

Advanced Powder Technology 21, 623 (2010).

${ }^{17}$ G. Juarez, P. Chen, and R. M. Lueptow, New Journal of Physics 13 (2011), 10.1088/1367-2630/13/5/053055.

${ }^{18}$ H. R. Norouzi, R. Zarghami, and N. Mostoufi, Chemical Engineering Research and Design 102, 12 (2015).

${ }^{19}$ GDR-MiDi, The European physical journal. E, Soft matter 14, 341 (2004), 0312502.

${ }^{20}$ F. Pignatel, C. Asselin, L. Krieger, I. C. Christov, J. M. Ottino, and R. M. Lueptow, Physical Review E - Statistical, Nonlinear, and Soft Matter Physics 86, 1 (2012).

${ }^{21}$ J. Mellmann, Powder Technology 118, 251 (2001).

${ }^{22}$ P. Jop, Y. Forterre, and O. Pouliquen, Nature 441, 727 (2006).

${ }^{23}$ P. P. Cortet, D. Bonamy, F. Daviaud, O. Dauchot, B. Dubrulle, and M. Renouf, Epl 88 (2009), 10.1209/0295-5075/88/14001, 0904.4679.

${ }^{24}$ L. F. Orozco, D.-H. Nguyen, J.-Y. Delenne, P. Sornay, and F. Radjai, Powder Technology 362, 157 (2020).

${ }^{25}$ Y. L. Ding, J. P. K. Seville, R. Forster, and D. J. Parker, Chemical Engineering Science 2509 (2001), 10.1016/S0009-2509(00)00468-1.

${ }^{26}$ A. A. Aissa, C. Duchesne, and D. Rodrigue, Powder Technology 219, 193 (2012).

${ }^{27}$ E. Alizadeh, O. Dubé, F. Bertrand, and J. Chaouki, AIChE Journal 59, 1894 (2013).

${ }^{28}$ S. Yang, A. Cahyadi, J. Wang, and J. W. Chew, AIChE Journal 62, 3874 (2016).

${ }^{29}$ G. Félix, V. Falk, and U. D’Ortona, European Physical Journal E 22, 25 (2007), 0604461.

${ }^{30}$ I. Govender, Minerals Engineering 92, 168 (2016).

${ }^{31}$ J. J. Moreau, European journal of mechanics. A. Solids 13, 93 (1994).

${ }^{32}$ F. Radjai and V. Richefeu, Mechanics of Materials 41, 715 (2009).

${ }^{33}$ G. Lois, A. Lemaître, and J. M. Carlson, Phys. Rev. E 72, 051303 (2005).

${ }^{34}$ P. Y. Lagrée, L. Staron, and S. Popinet, Journal of Fluid Mechanics 686, 378 (2011).

${ }^{35}$ M. Jean, V. Acary, and Y. Monerie, Philosophical Transactions of the Royal Society of London A: Mathematical, Physical and Engineering Sciences 359, 2497 (2001).

${ }^{36}$ J. O. Bird and P. J. Chivers, in Newnes Engineering and Physical Science Pocket Book, edited by J. O. Bird and P. J. Chivers (Newnes, 1993) pp. 235 - 237.

${ }^{37}$ H. Tang, R. Song, Y. Dong, and X. Song, Materials 12, 3170 (2019).

${ }^{38}$ L. Staron and E. J. Hinch, Journal of Fluid Mechanics 545, 1-27 (2005). 